\title{
FINITE GK-DIMENSIONAL PRE-NICHOLS ALGEBRAS OF QUANTUM LINEAR SPACES AND OF CARTAN TYPE
}

\author{
NICOLÁS ANDRUSKIEWITSCH AND GUILLERMO SANMARCO
}

\begin{abstract}
We study pre-Nichols algebras of quantum linear spaces and of Cartan type with finite GK-dimension. We prove that except for a short list of exceptions involving only roots of order 2, 3, 4, 6, any such pre-Nichols algebra is a quotient of the distinguished pre-Nichols algebra introduced by Angiono generalizing the De Concini-Kac-Procesi quantum groups. There are two new examples, one of which can be thought of as $G_{2}$ at a third root of one.
\end{abstract}

\section{Contents}

1. Introduction

2. Preliminaries

3. Quantum linear spaces

4. Cartan type

5. On the open cases

Acknowledgments

References

\section{INTRODUCTION}

\subsection{Overview.}

1.1.1. The problem. Let $\mathbb{k}$ be a field. Let GK-dim be an abbreviation of GelfandKirillov dimension, see $\mathrm{KL}$. In this paper we contribute to the ongoing program of classifying Hopf algebras with finite GK-dim. See $[\mathrm{B}+, \mathrm{G}, \mathrm{L}]$ and references therein.

Let $H$ be a Hopf algebra and let ${ }_{H}^{H} \mathcal{Y} \mathcal{D}$ be the category of Yetter-Drinfeld modules over $H$. Assume that $H$ is pointed (similar arguments apply more generally if its coradical is a Hopf subalgebra). Basic invariants of $H$ are

(i) the group of grouplikes $\Gamma=G(H)$,

(ii) the diagram $R=\oplus_{n \in \mathbb{N}_{0}} R^{n}$, a graded connected Hopf algebra in ${ }_{k \mathrm{k} \Gamma} \mathrm{Y} \mathcal{Y}$,

(iii) the infinitesimal braiding $V:=R^{1}$, an object in ${ }_{\mathbb{k} \Gamma}^{\mathrm{k} \Gamma} \mathcal{Y} \mathcal{D}$.

Received by the editors February 25, 2020, and, in revised form, September 29, 2020.

2020 Mathematics Subject Classification. Primary 16T20, 17 B37.

This material is based upon work supported by the National Science Foundation under Grant No. DMS-1440140 while the first author was in residence at the Mathematical Sciences Research Institute in Berkeley, California, in the Spring 2020 semester. The work of both authors was partially supported by CONICET, Secyt (UNC) and the Alexander von Humboldt Foundation through the Research Group Linkage Programme. 
See [AS3]. Assume that $\Gamma$ has finite growth. In order to classify those $H$ with finite GK-dim, one first needs to understand all such $R$ with finite GK-dim. As a coalgebra $R$ is coradically graded and connected; in other words, it is strictly graded as in $[\mathrm{Sw}$, Section 11.2, see p. 232 and Lemma 11.2.1]. Strictly graded Hopf algebras $R$ in ${ }_{k=\Gamma}^{k} \mathcal{Y} \mathcal{D}$ with $R^{1} \simeq V$ are called post-Nichols algebras of $V$; also, graded Hopf algebras $R$ in ${ }_{\mathbb{k} \Gamma}^{\mathbb{k} \Gamma} \mathcal{Y} \mathcal{D}$ generated by $R^{1} \simeq V$ are called pre-Nichols algebras of $V$. See 2.5 .

The Nichols algebra $\mathscr{B}(V)$ is isomorphic to the subalgebra of $R$ generated by $V$; see [A] for an introduction to Nichols algebras. When char $\mathbb{k}=0$ and $\operatorname{dim} H<\infty$ (thus $\Gamma$ is finite), it was conjectured in AS2 that $R=\mathscr{B}(V)$; this is known to be true when $\Gamma$ is abelian by An1. The validity of this conjecture says that the classification of the finite-dimensional Nichols algebras in ${ }_{k}{ }_{k} \Gamma \mathcal{Y} \mathcal{D}$ is a substantial step towards the problem of classifying finite-dimensional pointed Hopf algebras with group $\Gamma$. When char $\mathbb{k}>0$ or $\operatorname{dim} H=\infty$, the conjecture fails to be true and the knowledge of the Nichols algebras is not enough. Thus, towards classifying pointed Hopf algebras with group $\Gamma$ and finite GK-dim, we do not see how to avoid the consideration of the following questions:

(A) classify all $V \in{ }_{\mathbb{k} \Gamma}^{\mathbb{k} \Gamma} \mathcal{Y} \mathcal{D}$ such that $\mathscr{B}(V)$ has finite GK-dim,

(B) for such $V$ classify all post-Nichols algebras with finite GK-dim.

Question (B) appears to be difficult to handle directly. However Lemma 2.2 below, proved in AAH3, reduces Question (B) for $V$ as in (A) to

(C) classify all pre-Nichols algebras of $V^{*}$ with finite GK-dim.

As usual it is more flexible to deal with classes of braided vector spaces rather than classes of groups $\Gamma$ and correspondingly pre-Nichols algebras as braided Hopf algebras; see $§ 2.3$ for unexplained vocabulary.

1.1.2. Eminent pre-Nichols algebras. For Question $(\mathrm{C})$ we point out that all preNichols algebras of $V$ form a poset $\mathfrak{P r e}(V)$ with $T(V)$ minimal and $\mathscr{B}(V)$ maximal; those with finite GK-dim form a saturated subposet $\mathfrak{P r e}_{\mathrm{fGK}}(V)$, cf. \$2.5. When char $\mathbb{k}=0$ and the braiding is the usual flip, the Nichols algebra is just the symmetric algebra and the pre-Nichols algebras with finite GK-dim are the universal enveloping algebras of the finite-dimensional $\mathbb{N}$-graded Lie algebras generated in degree one. Thus $\mathfrak{P r e}_{\mathrm{fGK}}(V)$ is hardly computable when $\operatorname{dim} V \geq 2$. Similar considerations are valid when the braiding is the super flip of a super vector space, see 2.9.2. But if $\operatorname{dim} V=1$ and char $\mathbb{k}=0$, then $\mathfrak{P r e}_{\mathrm{fGK}}(V)=\mathfrak{P r e}(V)$ has obviously a minimal element. We introduce in this paper the notion of eminent pre-Nichols algebra as one that is a minimum in $\mathfrak{P r e}_{\mathrm{fGK}}(V)$. That is, a pre-Nichols algebra $\widehat{\mathscr{B}}$ of a braided vector $V$ is eminent if

(a) GK-dim $\widehat{\mathscr{B}}<\infty$;

(b) if $\mathscr{B}$ is a pre-Nichols algebra of $V$ with GK-dim $\mathscr{B}<\infty$, then there exists a morphism of pre-Nichols algebras $\widehat{\mathscr{B}} \rightarrow \mathscr{B}$, necessarily surjective.

The existence of an eminent pre-Nichols algebra $\widehat{\mathscr{B}}$ reduces Question $(\mathrm{C})$ to the determination of all pre-Nichols algebra quotients of $\widehat{\mathscr{B}}$, that is its homogeneous Hopf ideals starting in degree (at least) 2. Presently there is no general recipe to decide whether a braided vector space admits an eminent pre-Nichols algebra. In 
this paper we shall show that many braided vector spaces of diagonal type have eminent pre-Nichols algebras.

1.1.3. Distinguished pre-Nichols algebras. From now on we assume that $\mathbb{k}$ is algebraically closed and char $\mathbb{k}=0$. In this paper we deal with Question (C) for braided vector spaces $V$ of diagonal type, i.e. with braiding determined by a matrix $\mathfrak{q}=\left(q_{i j}\right)_{i, j \in \mathbb{I}}$ with entries in $\mathbb{k}^{\times}$where $\theta \in \mathbb{N}$ and $\mathbb{I}=\{1, \ldots, \theta\}$. See $\$ 2.8$ for precise definitions.

First we need to discuss Question (A) for this class. Finite-dimensional Nichols algebras of diagonal type, i.e. those with GK-dim $=0$, were classified in [H1] through the notion of (generalized) root system. More generally the list of all Nichols algebras of diagonal type with finite root system is given in loc. cit. It was conjectured in [AAH1, and verified in various cases $[\mathrm{R}$, AA1, AAH2, that Nichols algebras of diagonal type with finite GK-dim are precisely those with finite root system. We recall this as Conjecture 2.6. We shall assume in a few proofs that Conjecture 2.6 is valid in dimensions $\leq 5$.

Let $\mathscr{B}(V)=T(V) / \mathcal{J}(V)$ be a finite-dimensional Nichols algebra of diagonal type. The distinguished pre-Nichols algebra of $V$ introduced in [An3] is the quotient $\widetilde{\mathscr{B}}(V):=T(V) / \mathcal{I}(V)$, where $\mathcal{I}(V)$ is the ideal of $T(V)$ generated by the defining relations of $\mathcal{J}(V)$ given in An1 but excluding the powers of the Cartan root vectors and including the quantum Serre relations at Cartan vertices. Detailed presentations of $\mathcal{J}(V)$ and $\mathcal{I}(V)$ are available in [AA2, §4]. The notion of Cartan root requires the theory of Weyl groupoid that would led us too far from the goal of this paper. Indeed in Cartan type all roots are so and the distinguished pre-Nichols algebras are the positive parts of the quantum groups of [DKP]. Originally $\widetilde{\mathscr{B}}(V)$ was introduced as a tool for understanding the relations of $\mathscr{B}(V)$; several results on $\widetilde{\mathscr{B}}(V)$ were established in An3. The graded duals of the distinguished pre-Nichols algebras have been presented by generators and relations in AAR.

Unlike the notion of eminent pre-Nichols algebra, we lack at the moment a concise abstract definition of $\widetilde{\mathscr{B}}(V)$ that could be adapted beyond finite dimensional $\mathscr{B}(V)$ of diagonal type; but see $\$ 1.2 .1$ for quantum linear spaces.

In An3 the author and one of us asked whether a distinguished pre-Nichols algebra is eminent (in the terminology just introduced). Recall that the classification in [H1] was organized in [AA2 in various types: Cartan, standard, super, modular, super modular and UFO. Here we address the question above when $\mathscr{B}(V)$ is either a quantum linear space or of Cartan type. The recent paper ACS treats super and standard types, the remaining ones being the subject of work in progress.

1.2. The main results. In the present paper we focus on braided vector spaces of diagonal type of two kinds. Fix $V$ of diagonal type as in $\$ 2.8$, with braiding given by the matrix $\mathfrak{q}=\left(q_{i j}\right)_{i, j \in \mathbb{I}}$.

1.2.1. Quantum linear spaces. Here we assume that $\mathfrak{q}$ satisfies $q_{i j} q_{j i}=1$ for all $i \neq$ $j \in \mathbb{I}$. We extend the notion of distinguished pre-Nichols algebra to quantum linear spaces even when they are infinite-dimensional. Namely we define the distinguished pre-Nichols algebra $\widetilde{\mathscr{B}}(V)$ as the one presented by generators $\left(x_{i}\right)_{i \in \mathbb{I}}$ and relations $x_{i} x_{j}-q_{i j} x_{j} x_{i}$, for all $i \neq j \in \mathbb{I}$. If $1 \neq q_{i i}$ is a root of unit for all $i$, then $\operatorname{dim} \mathscr{B}(V)<\infty, V$ is of Cartan type $A_{1} \times \cdots \times A_{1}$ and any root is Cartan, so this definition is consistent with the one given in An3 and discussed above. 
We need some notation to state our first Theorem. Set

$$
\begin{array}{rlrl}
\mathbb{I}^{\infty} & =\left\{i \in \mathbb{I}: q_{i i} \notin \mathbb{G}_{\infty}\right\}, & \mathbb{I}^{N}=\left\{i \in \mathbb{I}: \text { ord } q_{i i}=N\right\}, N \geq 1, \\
\mathbb{I}^{t}=\bigcup_{N>3} \mathbb{I}^{N}, & \mathbb{I}^{ \pm}=\left\{i \in \mathbb{I}: q_{i i}= \pm 1\right\}=\mathbb{I}^{1} \sqcup \mathbb{I}^{2} .
\end{array}
$$

Thus $\mathbb{I}=\mathbb{I}^{ \pm} \sqcup \mathbb{I}^{3} \sqcup \mathbb{I}^{t} \sqcup \mathbb{I}^{\infty}$. For $\star \in \mathbb{N} \cup\{ \pm, t, \infty\}$, let $V^{\star}$ be the subspace of $V$ spanned by $\left(x_{i}\right)_{i \in \mathbb{I}^{\star}}$ and $\mathfrak{q}^{\star}$ the restriction of $\mathfrak{q}$ to $V^{\star}$. Then

$$
V=V^{ \pm} \oplus V^{3} \oplus V^{t} \oplus V^{\infty} \text {. }
$$

Theorem 1.1. Assume that Conjecture 2.6 is true.

(a) The distinguished pre-Nichols algebra $\widetilde{\mathscr{B}}\left(V^{\star}\right)$ is eminent, $\star \in\{3, t, \infty\}$.

(b) Let $\mathscr{B}$ be a finite GK-dimensional pre-Nichols algebra of $V$; let $\mathscr{B}^{ \pm, 3}$, respectively $\mathscr{B}^{t}, \mathscr{B}^{\infty}$ be the subalgebra of $\mathscr{B}$ generated by $V^{ \pm} \oplus V^{3}$, respectively $V^{t}$, $V^{\infty}$. Then there is a decomposition

$$
\mathscr{B} \simeq \mathscr{B}^{ \pm, 3} \underline{\otimes} \mathscr{B}^{t} \underline{\otimes} \mathscr{B}^{\infty} .
$$

(c) Assume that $V$ has a basis $\left\{x_{1}, x_{2}\right\}$ with $x_{1} \in V^{3}, x_{2} \in V^{1}$. Then

$$
\breve{\mathscr{B}}(V)=T(V) /\left\langle\left(\operatorname{ad}_{c} x_{1}\right)^{4}\left(x_{2}\right),\left(\operatorname{ad}_{c} x_{2}\right)^{2}\left(x_{1}\right)\right\rangle
$$

is an eminent pre-Nichols algebra of $V$ and has $\mathrm{GK}-\operatorname{dim}=6$.

See $\$ 2.1$ for the meaning of $\otimes$. Parts (a) and (b) follow from Proposition 3.2 whose proof assumes that Conjecture 2.6 is true. Part (c) is Proposition [3.3, Although $\breve{\mathscr{B}}(V)$ of part (c) is not the distinguished pre-Nichols algebra of the quantum plane $V$, it can be thought of as the distinguished one of the braided vector space of Cartan type $G_{2}$, but degenerated in the sense that the parameter is a primitive third root of unity. Via suitable bosonizations, $\breve{\mathscr{B}}(V)$ provides new examples of pointed Hopf algebras with finite GK-dim.

Let $\mathscr{B} \in \mathfrak{P r e}_{\mathrm{fGK}}(V)$. By $(\mathrm{a})$ and $(1.2)$ we have a surjective map of pre-Nichols algebras $\mathscr{B}^{ \pm, 3} \underline{\otimes} \widetilde{\mathscr{B}}\left(V^{t}\right) \underline{\otimes} \widetilde{\mathscr{B}}\left(V^{\infty}\right) \rightarrow \mathscr{B}$. Therefore it remains to understand $\mathscr{B}^{ \pm, 3}$. Towards this, we know:

- The pre-Nichols algebras of $V^{ \pm}$with finite GK-dim are (up to a twist) the enveloping superalgebras $U(\mathfrak{n})$, where $\mathfrak{n}=\oplus_{j \in \mathbb{N}} \mathfrak{n}^{j}$ is a finite-dimensional Lie superalgebra generated by $\mathfrak{n}^{1} \simeq V$, see $\oint_{2.9 .2}$.

- By Proposition $3.2, \mathscr{B}^{2,3} \simeq \mathscr{B}^{2} \underline{\otimes} \mathscr{B}^{3}$.

- For instance, if $V^{1}=0$, then there is a surjective map of pre-Nichols algebras $\mathscr{B}^{2} \underline{\otimes} \tilde{\mathscr{B}}\left(V^{3}\right) \underline{\otimes} \tilde{\mathscr{B}}\left(V^{t}\right) \underline{\otimes} \tilde{\mathscr{B}}\left(V^{\infty}\right) \rightarrow \mathscr{B}$.

Towards $\mathscr{B}^{1,3}$ we know Part (c) and $₫ 3.3$, It is natural to ask:

Question 1.2. Assume that $V=V^{1} \oplus V^{3}, \operatorname{dim} V^{1}=1$ and $\operatorname{dim} V^{3}=2$. Is the distinguished pre-Nichols algebra $\widetilde{\mathscr{B}}(V)$ eminent?

1.2.2. Connected Cartan type. Here $\mathfrak{q}$ is of finite Cartan type, i.e.

$$
q_{i j} q_{j i}=q_{i i}^{a_{i j}}, \quad \quad-\text { ord } q_{i i}<a_{i j} \leq 0, \quad i \neq j \in \mathbb{I},
$$

where $\mathbf{a}=\left(a_{i j}\right)_{i, j \in \mathbb{I}}$ is a Cartan matrix of finite type with connected Dynkin diagram. In 4 we recall the possibilities for such $\mathfrak{q}$. They depend on a root of unity $q$, whose order is denoted by $N$. In the following statement the symbols $x_{1112}, x_{2221}, x_{2112}, x_{1221}$ are defined in (2.1). 
Theorem 1.3.

(a) The distinguished pre-Nichols algebra $\widetilde{\mathscr{B}}_{\mathfrak{q}}$ is eminent except in the following cases: $A_{2}$ with $N=3$,

$$
A_{\theta}, \quad \theta \geq 2, \quad N=2 ; \quad D_{\theta}, \quad \theta \geq 4, \quad N=2 ; \quad G_{2}, \quad N=4,6 .
$$

(b) Suppose $\mathfrak{q}$ is of type $A_{2}$ with $N=3$. Then

$$
\widehat{\mathscr{B}}=\mathbb{k}\left\langle x_{1}, x_{2} \mid x_{1112}, x_{2221}, x_{2112}, x_{1221}\right\rangle
$$

is an eminent pre-Nichols algebra of $\mathfrak{q}$, and $\mathrm{GK}-\operatorname{dim} \widehat{\mathscr{B}}=5$.

This answers (partially) a question in An3.

The proof of (a) is given in Lemmas 4.12, 4.13, 4.15, 4.16, 4.17, 4.18, For the cases listed in (1.3) the determination of the poset $\mathfrak{P r e}_{\mathrm{fGK}}(V)$ remains an open problem except for $G_{2}$, with $N=4,6$ that was solved in [ACS]. See Section 5 for partial results; answers to Questions 5.2, 5.5, 5.7, 5.9 and 5.11 would shed light on the issue. The proof of (b) is given in Proposition 4.11, The eminent pre-Nichols algebra $\widehat{\mathscr{B}}$ is introduced and studied in $\$ 4.2 .2$ There we show that $\widehat{\mathscr{B}}$ properly covers the distinguished pre-Nichols algebra $\widetilde{\mathscr{B}}(V)$, which has GK-dim $\widetilde{\mathscr{B}}(V)=3$.

\section{Preliminaries}

2.1. Conventions. For $n \leq m \in \mathbb{N}_{0}$, put $\mathbb{I}_{n, m}=\left\{k \in \mathbb{N}_{0}: n \leq k \leq m\right\}$ and $\mathbb{I}_{m}=\mathbb{I}_{1, m}$. Given a positive integer $N$, we denote by $\mathbb{G}_{N}$ the group of $N$-th roots of unity in $\mathbb{k}^{\times}$, and by $\mathbb{G}_{N}^{\prime} \subset \mathbb{G}_{N}$ the subset of those of order $N$. The group of all roots of unity is denoted by $\mathbb{G}_{\infty}$ and $\mathbb{G}_{\infty}^{\prime}:=\mathbb{G}_{\infty}-\{1\}$.

The subalgebra generated by a subset $X$ of an associative algebra is denoted by $\mathbb{k}\langle X\rangle$.

All Hopf algebras are assumed to have bijective antipode. If $H$ is a Hopf algebra, the group of group-like elements is denoted by $G(H)$, while $\mathcal{P}(H)$ is the subspace of primitive elements. By gr $H$ we mean the graded coalgebra associated to the coradical filtration.

If $A$ and $B$ are algebras in ${ }_{H}^{H} \mathcal{Y} \mathcal{D}$, we denote by $A \underline{\otimes B}=\left(A \otimes B, \mu_{A \otimes B}\right)$ the algebra with multiplication $\mu_{A \otimes B}=\left(\mu_{A} \otimes \mu_{B}\right)\left(\operatorname{id}_{A} \otimes c_{B, A} \otimes \operatorname{id}_{B}\right)$, where $\mu_{A}$ and $\mu_{B}$ are the multiplications of $A$ and $B$, respectively.

2.2. Gelfand-Kirillov dimension. We refer to $\mathrm{KL}$ for general information on this topic. The following useful statement is immediate from the definition of GK-dim. Let $R$ be a ring and let $M=\oplus_{n \in \mathbb{N}_{0}} M_{n}$ be a direct sum of $R$-modules $M_{n}$ which are free of finite rank (we say $M$ is a locally finite graded $R$-module). The Poincaré series of $M$ is

$$
P_{M}:=\sum_{n \in \mathbb{N}_{0}} \operatorname{rank} M_{n} X^{n} \in \mathbb{Z}[[X]] .
$$

Lemma 2.1. Let $\mathbb{L}$ and $\mathbb{F}$ be fields and let $T=\oplus_{n \in \mathbb{N}_{0}} T_{n}$ and $U=\oplus_{n \in \mathbb{N}_{0}} U_{n}$ be two locally finite graded algebras generated in degree one over $\mathbb{L}$ and $\mathbb{F}$ respectively. If $P_{T}=P_{U}$, then GK-dim $T=$ GK-dim $U$.

Actually $[\mathrm{KL}, 12.6 .2]$ shows that the Poincaré series of a graded finitely generated algebra provides its GK-dim. 
2.3. Braided Hopf algebras. A pair $(V, c)$ where $V$ is a vector space and $c \in$ $G L\left(V^{\otimes 2}\right)$ satisfies the braid equation

$$
(c \otimes \mathrm{id})(\mathrm{id} \otimes c)(c \otimes \mathrm{id})=(\mathrm{id} \otimes c)(c \otimes \mathrm{id})(\mathrm{id} \otimes c)
$$

is called a braided vector space. A braided vector space with compatible algebra and coalgebra structures as in $[\mathrm{T}$ is called a braided Hopf algebra. For instance the tensor algebra $T(V)$ has a canonical structure of (graded connected) braided Hopf algebra such that the elements of degree 1 are primitive. Also the tensor coalgebra $T^{c}(V)$ becomes a braided Hopf algebra by the twisted shuffle product; see e.g. $[\mathrm{R}$, Proposition 9]. There is a homogeneous morphism of braided Hopf algebras $\Omega: T(V) \rightarrow T^{c}(V)$ determined by $\Omega(v)=v, v \in V$; its image is the Nichols algebra of $V$, denoted $\mathscr{B}(V)$. In fact $\Omega$ is the quantum symmetrizer, see e.g. A, Section $3.3]$.

Another description: let $\mathcal{J}(V)$ be the largest element of the set $\mathfrak{S}$ of graded Hopf ideals of $T(V)$ trivially intersecting $\mathbb{k} \oplus V$. Then $\mathscr{B}(V) \simeq T(V) / \mathcal{J}(V)$.

2.4. Principal realizations. Theorems 1.1 and 1.3 are relevant for the classification of Hopf algebras with finite GK-dim. Indeed a braided vector space arises (up to a mild condition) as a Yetter-Drinfeld module over a Hopf algebra; this is called a realization. Realizations are not unique and we single out a class of them for braidings of diagonal type. Let $H$ be a Hopf algebra. A YD-pair is a couple $(g, \chi) \subset G(H) \times \operatorname{Hom}_{\mathrm{Alg}}(H, \mathbb{k})$ satisfying

$$
\chi(h) g=\chi\left(h_{(2)}\right) h_{(1)} g \mathcal{S}\left(h_{(3)}\right), \quad h \in H .
$$

Compare with AS1, p. 671]. This compatibility guarantees that $\mathbb{k}_{g}^{\chi}$ (i. e. $H$ acting and coacting on $\mathbb{k}$ by $\chi$ and $g$, respectively) is a Yetter-Drinfeld module over $H$. Let $\left(V, c^{\mathfrak{q}}\right)$ be a braided vector space of diagonal type. Following [AS1, p. 673], a principal realization of $\left(V, c^{\mathfrak{q}}\right)$ over $H$ is a family $\left(g_{i}, \chi_{i}\right)_{i \in \mathbb{I}}$ of YD-pairs such that $q_{i j}=\chi_{j}\left(g_{i}\right)$ for all $i, j$. In this case $V=\bigoplus_{i} \mathbb{k}_{g_{i}}^{\chi_{i}} \in{ }_{H}^{H} \mathcal{Y D}$.

2.5. Pre-Nichols and post-Nichols algebras. We present in detail the objects of interest in this paper.

- Let $\mathscr{B}=\bigoplus_{n \in \mathbb{N}_{0}} \mathscr{B}^{n}$ be a graded connected braided Hopf algebra with $\mathscr{B}^{1} \simeq V$. Then $\mathscr{B}$ is a pre-Nichols algebra of $V$ if it is generated by $\mathscr{B}^{1}$. In this case there are epimorphisms of (graded) braided Hopf algebras

$$
T(V) \rightarrow \mathscr{B} \rightarrow \mathscr{B}(V) .
$$

Hence the set $\mathfrak{P r e}(V)$ of isomorphism classes of pre-Nichols algebras of $V$ is partially ordered with $T(V)$ minimal and $\mathscr{B}(V)$ maximal:

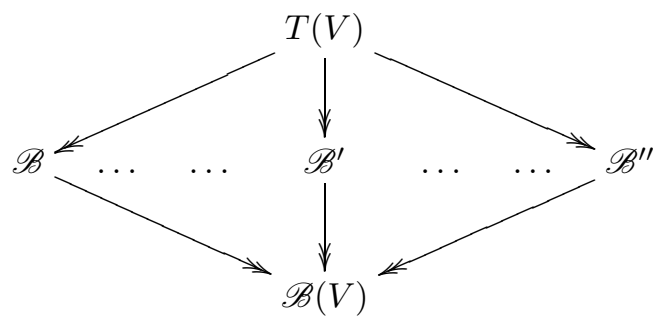


- Dually, a graded connected braided Hopf algebra $\mathcal{E}=\bigoplus_{n \in \mathbb{N}_{0}} \mathcal{E}^{n}$ with $\mathcal{E}^{1} \simeq V$ is a post-Nichols algebra of $V$ if it is coradically graded. Thus we have monomorphisms of (graded) braided Hopf algebras

$$
\mathscr{B}(V) \hookrightarrow \mathcal{E} \hookrightarrow T^{c}(V) .
$$

Hence the set $\mathfrak{P o s t}(V)$ of isomorphism classes of post-Nichols algebras of $V$ is partially ordered with $T^{c}(V)$ maximal and $\mathscr{B}(V)$ minimal:

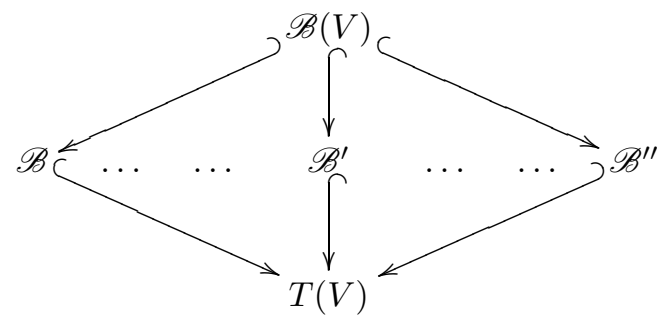

The only pre-Nichols which is also a post-Nichols algebra of $V$ is $\mathscr{B}(V)$ itself.

2.6. Eminent pre- and post-Nichols algebras. For the purposes of classifying Hopf algebras with finite GK-dim, it is important to describe the (partially ordered) subset $\mathfrak{P o s t}_{\mathrm{fGK}}(V)$ of $\mathfrak{P o s t}(V)$ consisting of post-Nichols algebras with finite GK-dim. In this paper we are mainly interested in the (partially ordered) subset $\mathfrak{P r e} \mathfrak{f a K}_{\mathrm{fGK}}(V)$ of $\mathfrak{P r e}(V)$ consisting of pre-Nichols algebras with finite GK-dim. The reason to start with this is given by the following result:

Lemma 2.2 ([AAH3]). Let $\mathscr{B}$ be a pre-Nichols algebra of $V$ and let $\mathcal{E}=\mathscr{B}^{d}$ be the graded dual of $\mathscr{B}$. Then $\mathrm{GK}-\operatorname{dim} \mathcal{E} \leq \mathrm{GK}-\operatorname{dim} \mathscr{B}$. If $\mathcal{E}$ is finitely generated, then the equality holds.

A first approximation to the determination of $\mathfrak{P o s t}_{\mathrm{fGK}}(V)$ and $\mathfrak{P r e}_{\mathrm{fGK}}(V)$ is through the following notion.

\section{Definition 2.3.}

(a) A pre-Nichols algebra $\widehat{\mathscr{B}}$ is eminent if it is the minimum of $\mathfrak{P r e}_{\mathrm{fGK}}(V)$; i. e. there is an epimorphism of braided Hopf algebras $\widehat{\mathscr{B}} \rightarrow \mathscr{B}$ that is the identity on $V$ for any $\mathscr{B} \in \mathfrak{P r e} \mathfrak{f G K}_{\mathrm{fGK}}(V)$.

(b) A post-Nichols algebra $\widehat{\mathcal{E}}$ is eminent if it is the maximum of $\mathfrak{P o s t}_{\mathrm{fGK}}(V)$; that is for any $\mathcal{E} \in \mathfrak{P o s t}_{\mathrm{fGK}}(V)$, there is a monomorphism of braided Hopf algebras $\mathcal{E} \hookrightarrow \widehat{\mathcal{E}}$ that is the identity on $V$.

Beware that there are braided vector spaces without eminent pre-Nichols algebras; e. g., if $\operatorname{dim} V>1$ and the braiding is the usual flip, then $\mathfrak{P r e}_{\mathrm{fGK}}(V)$ has infinite chains. An intermediate situation could be described as follows.

Definition 2.4. A family $\left(\widehat{\mathscr{B}}_{i}\right)_{i \in I} \subset \mathfrak{P r e}_{\mathrm{fGK}}(V)$ is eminent if

(a) for any $\mathscr{B} \in \mathfrak{P r e}_{\mathrm{fGK}}(V)$, there exists $i \in I$ and an epimorphism of braided Hopf algebras $\widehat{\mathscr{B}}_{i} \rightarrow \mathscr{B}$ that is the identity on $V$, and

(b) $\left(\widehat{\mathscr{B}}_{i}\right)_{i \in I}$ is minimal among the families in $\mathfrak{P r e}_{\mathrm{fGK}}(V)$ satisfying (a)

Eminent families of post-Nichols algebras are defined similarly. 
All the notions above about braided Hopf algebras related to braided vector spaces have a counterpart for Yetter-Drinfeld modules. Namely, suppose that $(V, c)$ is realized in ${ }_{H}^{H} \mathcal{Y D}$ for some Hopf algebra $H$. Then $\mathfrak{P r e}^{H}(V)$ is the subset of $\mathfrak{P r e}(V)$ of pre-Nichols algebras that belong to ${ }_{H}^{H} \mathcal{Y} \mathcal{D}$; similarly we have $\mathfrak{P r e} \mathfrak{e}_{\mathrm{fGK}}^{H}(V)$, $\mathfrak{P o s t}^{H}(V), \mathfrak{P o s t}_{\mathrm{fGK}}^{H}(V)$, and also $H$-eminent pre-Nichols or post-Nichols algebras.

2.7. The adjoint representation and $q$-brackets. Any Hopf algebra $R$ in ${ }_{H}^{H} \mathcal{Y} \mathcal{D}$ comes equipped with the (left) adjoint representation $\operatorname{ad}_{c}: R \rightarrow$ End $R$, given by

$$
\left(\operatorname{ad}_{c} x\right) y=\mu(\mu \otimes \mathcal{S})(\mathrm{id} \otimes c)(\Delta \otimes \mathrm{id})(x \otimes y), \quad x, y \in R,
$$

where $\mu, \Delta$ and $\mathcal{S}$ denote the multiplication, comultiplication and antipode of $R$, respectively. The adjoint action of a primitive element $x \in R$ is

$$
\left(\operatorname{ad}_{c} x\right) y=x y-\left(x_{(-1)} \cdot y\right) x_{(0)}, \quad y \in R .
$$

Given $x_{i_{1}}, x_{i_{2}} \ldots, x_{i_{k}} \in R$, put

$$
x_{i_{1} i_{2} \ldots i_{k}}=\left(\operatorname{ad}_{c} x_{i_{1}}\right) \ldots\left(\operatorname{ad}_{c} x_{i_{k-1}}\right) x_{i_{k}} .
$$

We also set $x_{(k h)}=x_{k(k+1)(k+2) \ldots h}$ for $k<h$.

On the other hand, the braided commutator is defined by

$$
[x, y]_{c}=x y-\left(x_{(-1)} \cdot y\right) x_{(0)}, \quad x, y \in R .
$$

We refer to [AA2, Introduction] for a more detailed treatment.

2.8. Nichols algebras of diagonal type. Fix a natural number $\theta$ and let $\mathbb{I}=\mathbb{I}_{\theta}$. Any matrix $\mathfrak{q}=\left(q_{i j}\right)_{i, j \in \mathbb{I}}$ with coefficients in $\mathbb{k}^{\times}$determines a braided vector space of diagonal type $\left(V, c^{\mathfrak{q}}\right)$, where

$$
V \text { has a basis }\left(x_{i}\right)_{i \in \mathbb{I},} \quad c^{\mathfrak{q}}\left(x_{i} \otimes x_{j}\right)=q_{i j} x_{j} \otimes x_{i}, \quad i, j \in \mathbb{I} .
$$

The Dynkin diagram associated to $\mathfrak{q}$ is a non-oriented graph with $\theta$ vertices. The vertex $i$ is labelled by $q_{i i}$, and there is an edge between $i$ and $j$ if and only if $\widetilde{q}_{i j}:=q_{i j} q_{j i} \neq 1$; in this case, the edge is labeled by $\widetilde{q}_{i j}$. Thus we may speak of the connected components of this diagram and by abuse of notation of $\mathfrak{q}$. The following useful result says that a connected component with at least 2 vertices one of them labelled by 1 gives rise to an infinite GK-dimensional Nichols algebra.

Lemma 2.5 ([AAH1, Lemma 2.8]). Let $U$ be a braided vector space of diagonal type with Dynkin diagram

$$
\stackrel{q}{\circ} \longrightarrow \stackrel{r}{\circ}, \quad r \neq 1 .
$$

Then GK-dim $\mathscr{B}(U)=\infty$.

Let $\alpha_{1}, \ldots, \alpha_{\theta}$ be the canonical basis of $\mathbb{Z}^{\theta}$. From the braiding matrix $\mathfrak{q}$ we obtain a $\mathbb{k}^{\times}$-valued bilinear form on $\mathbb{Z}^{\theta}$, still denoted $\mathfrak{q}$ and determined by $\mathfrak{q}\left(\alpha_{i}, \alpha_{j}\right)=q_{i j}$, $i, j \in \mathbb{I}$. Put also

$$
\widetilde{\mathfrak{q}}(\alpha, \beta):=\mathfrak{q}(\alpha, \beta) \mathfrak{q}(\beta, \alpha), \quad \alpha, \beta \in \mathbb{Z}^{\theta} .
$$

For sake of brevity, we use $\mathfrak{q}_{\alpha \beta}=\mathfrak{q}(\alpha, \beta)$ and $\widetilde{\mathfrak{q}}_{\alpha \beta}=\widetilde{\mathfrak{q}}(\alpha, \beta)$ as well.

The braided vector space $\left(V, c^{\mathfrak{q}}\right)$ as in (2.2) is realized in ${ }_{\mathbb{Z}^{\theta}}^{\mathbb{Y}^{\theta} \mathcal{D}}$ by declaring

$$
\operatorname{deg}\left(x_{i}\right)=\alpha_{i}, \quad \alpha_{i} \cdot x_{j}=q_{i j} x_{j}, \quad i, j \in \mathbb{I} .
$$


The algebra $T(V)$ becomes $\mathbb{Z}^{\theta}$-graded. Thus any quotient algebra $R$ of $T(V)$ by a graded ideal inherits the grading: $R=\bigoplus_{\alpha \in \mathbb{Z}^{\theta}} R^{\alpha}$. We keep the notation deg for this degree. Furthermore, if $R$ is an algebra obtained as a quotient of $T(V)$ by a graded ideal $I$ (thus a subobject in ${\underset{\mathbb{Z}}{\theta}}^{\theta} \mathcal{Y} \mathcal{D}$ ), then the braiding on the homogeneous subspaces is given by

$$
c(u \otimes v)=\mathfrak{q}_{\alpha, \beta} v \otimes u, \quad u \in R^{\alpha}, v \in R^{\beta} .
$$

We shall use (2.5) many times. The braided commutators satisfy

$$
\begin{aligned}
{[u, v w]_{c} } & =[u, v]_{c} w+\mathfrak{q}_{\alpha \beta} v[u, w]_{c}, \\
{[u v, w]_{c} } & =\mathfrak{q}_{\beta \gamma}[u, w]_{c} v+u[v, w]_{c}, \\
{\left[[u, v]_{c}, w\right]_{c} } & =\left[u,[v, w]_{c}\right]_{c}-\mathfrak{q}_{\alpha \beta} v[u, w]_{c}+\mathfrak{q}_{\beta \gamma}[u, w]_{c} v,
\end{aligned}
$$

for homogeneous elements $u \in R^{\alpha}, v \in R^{\beta}, w \in R^{\gamma}$.

In the diagonal setting (2.2) we set as usual $\mathcal{J}_{\mathfrak{q}}=\mathcal{J}(V), \mathscr{B}_{\mathfrak{q}}=\mathscr{B}(V), \widetilde{\mathscr{B}}_{\mathfrak{q}}=$ $\widetilde{\mathscr{B}}(V)$, etc. Nichols algebras of diagonal type (i. e. those arising from braided vector spaces of diagonal type) have been intensively studied. The classification of all matrices $\mathfrak{q}$ such that $\mathscr{B}_{\mathfrak{q}}$ has finite root system was provided in [H1] the defining relations of these Nichols algebras are given in An1, An2. Clearly, finite dimensional Nichols algebras of diagonal type have finite root system. It was conjectured that those of finite GK-dim share the same property.

Conjecture 2.6 ([AAH1, Conjecture 1.5]). The root system of a Nichols algebra of diagonal type with finite GK-dimension is finite.

The validity of Conjecture 2.6 would imply the classification of finite GK-dimensional Nichols algebras of diagonal type. There is strong evidence supporting it. The conjecture holds when $\theta=2$ AAH2, Thm. 4.1], when the braiding is of affine Cartan type [AAH2, Thm. 1.2], or when $\mathfrak{q}$ is generic, that is $q_{i i} \notin \mathbb{G}_{\infty}$, and $q_{i j} q_{j i}=1$ or $q_{i j} q_{j i} \notin \mathbb{G}_{\infty}$, for all $i \neq j \in \mathbb{I}[\mathrm{R}, \mathrm{AA} 1]$.

We include for completeness proofs of the following well-known results.

Lemma 2.7. Let $0 \neq v, w \in T(V)$ be homogeneous primitive elements with $\operatorname{deg} v=$ $\alpha$ and $\operatorname{deg} w=\beta$. Then $\left(\operatorname{ad}_{c} v\right) w$ is primitive if and only if $\widetilde{\mathfrak{q}}_{\alpha \beta}=1$.

Proof. Using (2.5), compute $\Delta\left(\left(\operatorname{ad}_{c} v\right) w\right)=\Delta\left(v w-\mathfrak{q}_{\alpha \beta} w v\right)=$

$$
\begin{aligned}
= & (v \otimes 1+1 \otimes v)(w \otimes 1+1 \otimes w)-\mathfrak{q}_{\alpha \beta}(w \otimes 1+1 \otimes w)(v \otimes 1+1 \otimes v) \\
= & v w \otimes 1+v \otimes w+\mathfrak{q}_{\alpha \beta} w \otimes v+1 \otimes v w \\
& -\mathfrak{q}_{\alpha \beta}\left(w v \otimes 1+w \otimes v+\mathfrak{q}_{\beta \alpha} v \otimes w+1 \otimes w v\right) \\
= & \left(\operatorname{ad}_{c} v\right) w \otimes 1+1 \otimes\left(\operatorname{ad}_{c} v\right) w+\left(1-\widetilde{\mathfrak{q}}_{\alpha \beta}\right) v \otimes w .
\end{aligned}
$$

Lemma 2.8. Let $R$ be a graded braided Hopf algebra. If $W$ is any braided subspace of $R$ contained in $\mathcal{P}(R)$ then $\mathrm{GK}-\operatorname{dim} \mathscr{B}(W) \leq \mathrm{GK}-\operatorname{dim} R$.

Proof. We follow [AS4, Lemma 5.4]. Since the elements of $W$ are primitive, the subalgebra $\mathbb{k}\langle W\rangle$ is a braided Hopf subalgebra of $R$; by definition of the Nichols algebra it follows that $\operatorname{gr} \mathbb{k}\langle W\rangle$ projects onto $\mathscr{B}(W)$, so GK-dim $\mathscr{B}(W) \leq \mathrm{GK}$-dim gr $\mathbb{k}\langle W\rangle$. But GK-dim $\operatorname{gr} \mathbb{k}\langle W\rangle \leq \mathrm{GK}-\operatorname{dim} \mathbb{k}\langle W\rangle$ by [KL, Lemma 6.5], and this proves the desired inequality. 
2.9. Pre-Nichols algebras of diagonal type. Let $\left(V, c^{\mathfrak{q}}\right)$ be a braided vector space of diagonal type associated to the matrix $\mathfrak{q}=\left(q_{i j}\right)_{i, j \in \mathbb{I}}$. Recall that $\widetilde{q}_{i j}=$ $q_{i j} q_{j i}, i \neq j$. We write $\mathfrak{P r e} \mathfrak{Z}_{\mathrm{fGK}}^{\mathbb{Z}^{\theta}}(V)$ for $\mathfrak{P r} \mathfrak{e}_{\mathrm{fGK}}^{\mathrm{k} \mathbb{Z}^{\theta}}(V)$, cf. (2.4).

2.9.1. Pre-Nichols algebras under twist-equivalence. Let $\mathfrak{p}=\left(p_{i j}\right)_{i, j \in \mathbb{I}}$ be another braiding matrix such that

$$
q_{i i}=p_{i i}, \quad \quad \widetilde{q}_{i j}=\widetilde{p}_{i j}, \quad i, j \in \mathbb{I} .
$$

In this case, $\left(V, c^{\mathfrak{q}}\right)$ and the braided vector space $\left(W, c^{\mathfrak{p}}\right)$ with basis $\left(y_{i}\right)_{i \in \mathbb{I}}$ are said to be twist-equivalent.

Lemma 2.9. There is an isomorphism of posets $\mathfrak{P r e}_{\mathrm{fGK}}^{\mathbb{Z}^{\theta}}(W) \simeq \mathfrak{P r e} \mathfrak{f G K}_{\mathrm{fGK}}^{\mathbb{Z}^{\theta}}(V)$.

Proof. Let $\sigma: \mathbb{Z}^{\theta} \times \mathbb{Z}^{\theta} \rightarrow \mathbb{k}^{\times}$be the bilinear form, hence a 2-cocycle, given by $\sigma\left(\alpha_{i}, \alpha_{j}\right)=\left\{\begin{array}{ll}p_{i j} q_{i j}^{-1}, & i \leq j, \\ 1, & i>j\end{array}\right.$. Let $T(V)_{\sigma}$ be the corresponding cocycle deformation of $T(V)$, i. e. with multiplication

$$
u \cdot \sigma v=\sigma(\alpha, \beta) u v, \quad u \in T(V)^{\alpha}, v \in T(V)^{\beta}, \alpha, \beta \in \mathbb{Z}^{\theta} .
$$

By the proof of [AS3, Prop. 3.9] the linear map $\varphi: W \rightarrow V, \varphi\left(y_{i}\right)=x_{i}, i \in \mathbb{I}$, induces an isomorphism $\varphi: T(W) \rightarrow T(V)_{\sigma}$ of Hopf algebras in $\mathbb{Z}^{\theta} \mathcal{Y} \mathcal{D}$. Let $I$ be a Hopf ideal of $T(V)$ that belongs to $\mathbb{Z}^{\theta} \mathcal{Y} \mathcal{D}$; then it is also a Hopf ideal of $T(V)_{\sigma}$ and $\mathrm{GK}-\operatorname{dim} T(V) / I=\mathrm{GK}-\operatorname{dim} T(V)_{\sigma} / I$ by Lemma 2.1

2.9.2. Pre-Nichols algebras of super symmetric algebras. Assume that $\widetilde{q}_{i j}=1=q_{i i}^{2}$, for all $j \neq i \in \mathbb{I}$. Then $V=V_{0} \oplus V_{1}$ is a super vector space where $V_{j}$ is spanned by those $x_{i}$ 's such that $q_{i i}=(-1)^{j}, j=0,1$. Let $\mathfrak{p}=\left(p_{i j}\right)_{i, j \in \mathbb{I}}$ be the matrix corresponding to the associated super symmetry. Then

- The pre-Nichols algebras of $\left(V, c^{\mathfrak{p}}\right)$ are the enveloping superalgebras $U(\mathfrak{n})$, where $\mathfrak{n}=\oplus_{j \in \mathbb{N}} \mathfrak{n}^{j}$ is a graded Lie superalgebra generated by $\mathfrak{n}^{1} \simeq V$.

- $\mathfrak{P r} \mathfrak{r}_{\mathrm{fGK}}\left(V, c^{\mathfrak{p}}\right)$ consists of the enveloping superalgebras $U(\mathfrak{n})$, where $\mathfrak{n}=\oplus_{j \in \mathbb{N}} \mathfrak{n}^{j}$ is a graded Lie superalgebra generated by $\mathfrak{n}^{1} \simeq V$ with $\operatorname{dim} \mathfrak{n}<\infty$.

- Hence $\mathfrak{P r e}_{\mathrm{fGK}}^{\mathbb{Z}^{\theta}}\left(V, c^{\mathfrak{p}}\right)$ consists of the enveloping superalgebras $U(\mathfrak{n})$, where $\mathfrak{n}=$ $\oplus_{\beta \in \mathbb{Z}^{\theta}} \mathfrak{n}^{\beta}$ is a finite-dimensional $\mathbb{Z}^{\theta}$-graded Lie superalgebra generated by $\mathfrak{n}^{1}=$ $\oplus_{i \in \mathbb{I}} \mathfrak{n}^{\alpha_{i}} \simeq V$. In particular $\mathfrak{P r e} \mathfrak{Z}_{\mathrm{fGK}}^{\mathbb{Z}^{\theta}}\left(V, c^{\mathfrak{p}}\right) \subsetneq \mathfrak{P} \mathfrak{r e}_{\mathrm{fGK}}\left(V, c^{\mathfrak{p}}\right)$.

- By Lemma 2.9, $\mathfrak{P r e}_{\mathrm{fGK}}^{\mathbb{Z}^{\theta}}\left(V, c^{\mathfrak{q}}\right)$ is isomorphic as a poset to the set of isomorphism classes of finite-dimensional $\mathbb{Z}^{\theta}$-graded Lie superalgebras as in the previous point.

\section{Quantum Linear SPaCes}

In this section we investigate finite GK-dimensional pre-Nichols algebras of quantum linear spaces. These are Nichols algebras of braided vector spaces of diagonal type with totally disconnected Dynkin diagram. More precisely, fix a matrix $\mathfrak{q}=\left(q_{i j}\right)_{i, j \in \mathbb{I}}$ and a vector space $V$ with basis $\left(x_{i}\right)_{i \in \mathbb{I}}$ and braiding given by $c^{\mathfrak{q}}\left(x_{i} \otimes x_{j}\right)=q_{i j} x_{j} \otimes x_{i}, i, j \in \mathbb{I}$. In this section we assume that

$$
q_{i j} q_{j i}=1, \quad i \neq j \in \mathbb{I} .
$$


Then $\mathscr{B}_{\mathfrak{q}}$ is presented by generators $\left(x_{i}\right)_{i \in \mathbb{I}}$ and relations

$$
\begin{aligned}
x_{i j}=0, & \text { if } i<j, \\
x_{i}^{N_{i}}=0, & \text { if } q_{i i} \in \mathbb{G}_{\infty}^{\prime},
\end{aligned}
$$$$
\text { where } N_{i}:=\operatorname{ord} q_{i i} \in \mathbb{N} \cup \infty \text {; }
$$

here we are using the notation (2.1). It has a PBW-basis:

$$
\left\{x_{1}^{a_{1}} x_{2}^{a_{2}} \cdots x_{\theta}^{a_{\theta}}: 0 \leq a_{i}<N_{i} \text { if } q_{i i} \in \mathbb{G}_{\infty}^{\prime} ; 0 \leq a_{i} \text { otherwise }\right\} .
$$

As defined in the Introduction, the distinguished pre-Nichols algebra $\widetilde{\mathscr{B}}_{\mathfrak{q}}$ of $V$ is presented by generators $\left(x_{i}\right)_{i \in \mathbb{I}}$ and relations (3.2); it is a domain of GK-dim $\theta$. Recall the partition $\mathbb{I}=\mathbb{I}^{ \pm} \sqcup \mathbb{I}^{3} \sqcup \mathbb{I}^{t} \sqcup \mathbb{I}^{\infty}$ where as in (1.1) we set

$$
\begin{array}{ll}
\mathbb{I}^{\infty}=\left\{i \in \mathbb{I}: q_{i i} \notin \mathbb{G}_{\infty}\right\}, & \mathbb{I}^{ \pm}=\left\{i \in \mathbb{I}: q_{i i}= \pm 1\right\}, \\
\mathbb{I}^{N}=\left\{i \in \mathbb{I}: q_{i i} \in \mathbb{G}_{N}^{\prime}\right\}, N \geq 3, & \mathbb{I}^{t}=\bigcup_{N>3} \mathbb{I}^{N} .
\end{array}
$$

For $\star \in\{ \pm, 3, t, \infty\}$, let $V^{\star}$ be the subspace of $V$ spanned by $\left(x_{i}\right)_{i \in \mathbb{I}^{\star}}$ and $\mathfrak{q}^{\star}$ the restriction of $\mathfrak{q}$ to $V^{\star}$. Then $V=V^{ \pm} \oplus V^{3} \oplus V^{t} \oplus V^{\infty}$. As we have seen in 2.9 .2 the $\mathbb{Z}^{\theta}$-graded pre-Nichols algebras of $V^{ \pm}$are twistings of enveloping algebras of nilpotent Lie superalgebras with suitable properties; in particular, there is no eminent pre-Nichols algebra of $V^{ \pm}$.

3.1. Reduction to order $\leq 3$. Below we consider various braided vector spaces of diagonal type, see 2.8 for the recipe of the Dynkin diagram that encodes the matrix $\mathfrak{q}$ that determines the braiding.

Remark 3.1. Let $i \neq j \in \mathbb{I}$. Recall that $x_{i j}:=\left(\operatorname{ad}_{c} x_{i}\right) x_{j}=x_{i} x_{j}-q_{i j} x_{j} x_{i}$. The braiding of the 3 -dimensional subspace $\mathbb{k} x_{i}+\mathbb{k} x_{i j}+\mathbb{k} x_{j} \subset T(V)$ is easily computed, and the corresponding Dynkin diagram is either

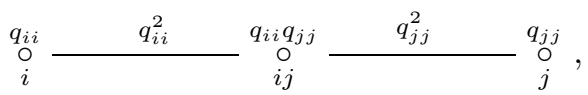

or it is disconnected if the label of some edge is 1 . Indeed,

$$
q_{i, i j}=q_{i i} q_{i j}, \quad q_{i j, i}=q_{i i} q_{j i}, \quad q_{i j, i j}=q_{i i} \widetilde{q}_{i j} q_{j j}, \quad q_{i j, j}=q_{i j} q_{j j}, \quad q_{j, i j}=q_{j i} q_{j j}
$$

so $\widetilde{q}_{i, i j}=q_{i i}^{2} \widetilde{q}_{i j}$ and $\widetilde{q}_{i j, j}=q_{j j}^{2} \widetilde{q}_{i j}$. Since $\widetilde{q}_{i j}=1$ because we are in the quantum linear space situation, (3.5) is the Dynkin diagram of $\mathbb{k} x_{i}+\mathbb{k} x_{i j}+\mathbb{k} x_{j}$.

Proposition 3.2. Let $i, j \in \mathbb{I}$ such that $4<\operatorname{ord} q_{i i}+\operatorname{ord} q_{j j}$. Assume that Conjecture 2.6 is true. Then $x_{i j}=0$ holds in any finite GK-dimensional pre-Nichols algebra of $\mathfrak{q}$.

We point out that Conjecture 2.6 is needed only to discard cases (11), (5) and (8) below, that require the conjecture only for dimension 3 .

Proof. Let $\mathscr{B}$ be a pre-Nichols algebra of $\mathfrak{q}$, so there is a braided Hopf algebra map $T(V) \rightarrow \mathscr{B}$. Let $y_{1}, y_{2}, y_{3}$ denote the image of $x_{i}, x_{j}, x_{i j}$, respectively, and consider $W:=\mathbb{k} y_{1}+\mathbb{k} y_{2}+\mathbb{k} y_{3}$. By Lemma 2.7 we have $W \subset \mathcal{P}(\mathscr{B})$, hence Lemma 2.8 warranties GK-dim $\mathscr{B}(W) \leq \mathrm{GK}$-dim $\mathscr{B}$.

Assume $y_{3} \neq 0$, so $W$ is 3 -dimensional by a degree argument and its Dynkin diagram $\mathfrak{D}$ is (3.5). We show that GK-dim $\mathscr{B}(W)=\infty$. 
Consider the subspaces $V_{1}=\mathbb{k} y_{1} \oplus \mathbb{k} y_{3}, V_{2}=\mathbb{k} y_{3} \oplus \mathbb{k} y_{2} \subset W$; denote their corresponding Dynkin diagrams by $\mathfrak{D}_{1}$ and $\mathfrak{D}_{2}$, respectively. From $q_{i j} q_{j i}=1$ it follows $x_{i j}=-q_{i j} x_{j i}$, so the image of $x_{j i}$ in $\mathscr{B}$ is not zero.

We split the proof in several cases according to the possibilities for ord $q_{i i}$ and ord $q_{j j}$.

Case $1\left(q_{i i} \notin \mathbb{G}_{\infty}\right.$ or $\left.q_{j j} \notin \mathbb{G}_{\infty}\right)$. This essentially goes back to $[\mathrm{R}$. Assume first $q_{i i} \notin \mathbb{G}_{\infty}$. If GK-dim $\mathscr{B}<\infty$, it follows from [AAH1, Lemmas 2.6 and 2.7] that there exists a natural number $k$ such that $(k)_{q_{i i}}^{!} \prod_{h=0}^{k-1}\left(1-q_{i i}^{h}\right)=0$, which contradicts $q_{i i} \notin \mathbb{G}_{\infty}$. The case $q_{j j} \notin \mathbb{G}_{\infty}$ is similar: since the image of $x_{j i}$ is not zero, we may apply the same argument as with $q_{i i}$.

Case $2\left(q_{i i}=1\right.$ or $\left.q_{j j}=1\right)$. We may suppose $q_{j j}=1$; if $q_{i i}=1$, the same argument applies. By the previous case, we may assume $q_{i i}$ is a root of unity, and by hypothesis its order must be $N_{i}>3$. The diagram $\mathfrak{D}_{1}$ is

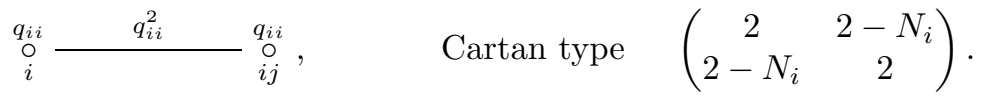

If GK-dim $\mathscr{B}\left(V_{1}\right)<\infty$ then AAH2 implies that the Cartan matrix is of finite type. Thus we conclude $N_{i}=3$, a contradiction.

Case $3\left(q_{i i}=1\right.$ or $\left.q_{j j}=-1\right)$. Assume that $q_{j j}=1$. By Case 1 , we may assume that $q_{i i}$ is a root of unity; by hypothesis, its order is $\geq 3$. By AAH2, GK-dim $\mathscr{B}\left(V_{1}\right)=$ $\infty$ since the Dynkin diagram of $V_{1}$ is

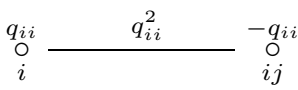

and this does not appear in [H1, Table 1]; indeed it is of Cartan, but not finite, type. The case $q_{i i}=-1$ is treated similarly.

Case $4\left(q_{i i}, q_{j j} \in \mathbb{G}_{\infty}-\mathbb{G}_{2}\right)$. Now $W$ has connected Dynkin diagram

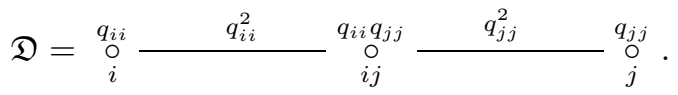

If the Nichols algebra of $V_{1}$ is finite GK-dimensional, by exhaustion of [H1, Table 1] we conclude that $q_{i i}, q_{j j}$ and $\mathfrak{D}_{1}$ satisfy one of the following:

(1) $q_{i i} \in \mathbb{G}_{3}^{\prime}, q_{i i} q_{j j}=-1, \quad \underset{i}{\stackrel{q_{i i}}{\circ}} \stackrel{q_{i i}^{2}}{i} \stackrel{-1}{\circ}$

(2) $q_{i i} \in \mathbb{G}_{4}^{\prime}, q_{j j}=q_{i i}, \underset{i}{\stackrel{q_{i i}}{\stackrel{\circ}{i}}-1} \stackrel{-1}{\stackrel{-1}{\circ}}$

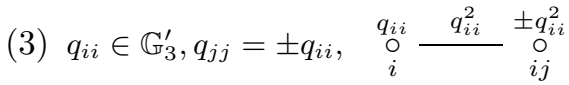

(4) $q_{i i} \in \mathbb{G}_{3}^{\prime}, q_{j j}=q_{i i}^{2}, \underset{i}{\stackrel{q_{i i}}{\circ}} \stackrel{q_{i i}^{2}}{\stackrel{1}{\circ}} \underset{i j}{\stackrel{1}{i}}$
(5) $q_{i i} \in \mathbb{G}_{6}^{\prime}, q_{i i} q_{j j}=-1, \quad \underset{i}{\stackrel{q_{i i}}{\circ}} \stackrel{q_{i i}^{2}}{\substack{-1 \\ i j}}$

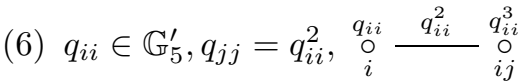

(7) $q_{j j} \in \mathbb{G}_{9}^{\prime}, q_{i i}=q_{j j}^{3}, \underset{i}{\stackrel{q_{j j}^{3}}{0}} \stackrel{q_{j j}^{6}}{q_{j j}^{4}} \underset{i j}{0}$

(8) $q_{i i} \in \mathbb{G}_{5}^{\prime}, q_{i i} q_{j j}=-1, \quad \begin{gathered}q_{i i} \\ \stackrel{i}{i}\end{gathered} \stackrel{q_{i i}^{2}}{-1} \begin{gathered}-1 \\ i j\end{gathered}$

In the rest of the proof, we discard one by one all these possibilities. 
(2) Now $W$ is of Cartan type with Dynkin diagram and Cartan matrix:

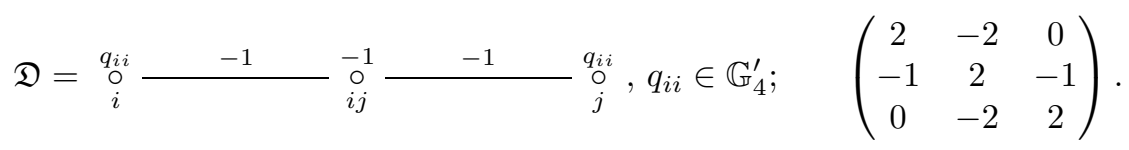

Since this matrix is of affine type, GK- $\operatorname{dim} \mathscr{B}(W)=\infty$ by [AH2].

(3) Assume first $q_{j j}=-q_{i i}$. Then

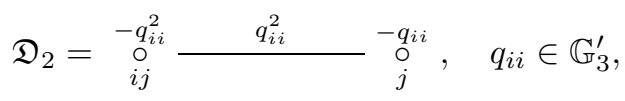

which is not arithmetic. By AAH2] we see that GK-dim $\mathscr{B}\left(V_{2}\right)=\infty$. Next, when $q_{j j}=q_{i i}, W$ is of Cartan type with Dynkin diagram and Cartan matrix:

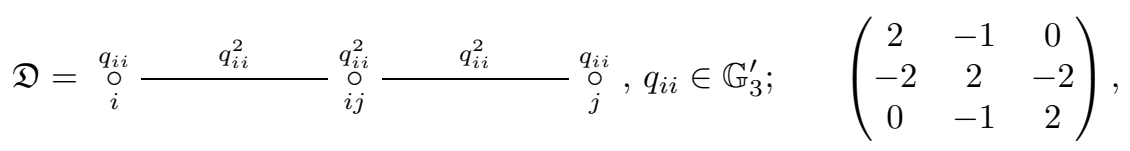

which is affine, so GK-dim $\mathscr{B}(W)=\infty$ by [AAH2] (4) Since $q_{i i}^{2} \neq 1$, we have GK- $\operatorname{dim} \mathscr{B}\left(V_{1}\right)=\infty$ by [AAH1, Lemma 2.8]. (6) In this case

$$
\mathfrak{D}_{2}=\underset{i j}{\stackrel{q_{i i}^{3}}{o}} \underset{i j}{q_{i i}^{4}} \stackrel{\substack{q_{i i}^{2} \\ j}}{\underset{j}{2}}, \quad q_{i i} \in \mathbb{G}_{5}^{\prime},
$$

is of indefinite Cartan type, so GK- $\operatorname{dim} \mathscr{B}\left(V_{2}\right)=\infty$ by [AAH2 . (7) Similarly,

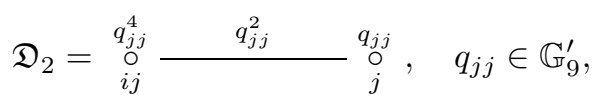

is indefinite Cartan, so GK- $\operatorname{dim} \mathscr{B}\left(V_{2}\right)=\infty$. In the remaining cases, $\mathfrak{D}$ is

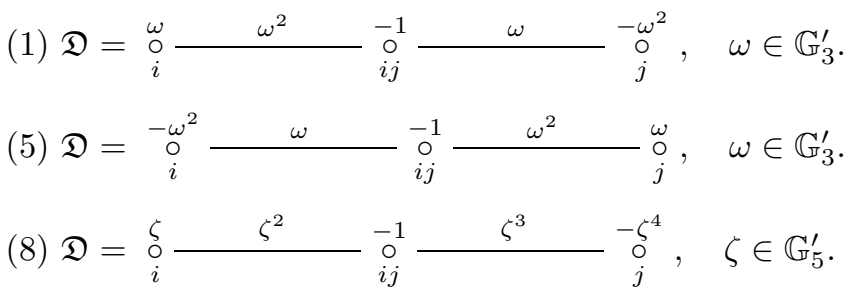

Now (11) and (5) are equal up to permutation of the indexes. Only here we need to assume the validity of Conjecture 2.6. Indeed, these diagrams do not appear in [H1, Table 2], so GK-dim $\mathscr{B}(W)=\infty$ in all cases.

3.2. A pre-Nichols algebra of type $G_{2}$. We assume $\left(V, c^{\mathfrak{q}}\right)$ has the following Dynkin diagram

$$
\begin{array}{lll}
\omega & 1 \\
0 & \stackrel{1}{0} \\
1 & 2
\end{array}, \quad \omega \in \mathbb{G}_{3}^{\prime} .
$$

Proposition 3.3. The algebra $\breve{\mathscr{B}}_{\mathfrak{q}}:=T(V) /\left\langle x_{11112}, x_{221}\right\rangle$ is an eminent preNichols algebra of $\left(V, c^{\mathfrak{q}}\right)$ and GK-dim $\breve{\mathscr{B}}_{\mathfrak{q}}=6$.

Proof. We first claim that the elements $x_{11112}$ and $x_{221}$ are primitive in $T(V)$. This is verified by a direct computation, see $[\underline{\mathrm{S}}$.

Second, we claim that the relations $x_{11112}=0$ and $x_{221}=0$ hold in any finite GK-dimensional pre-Nichols algebra $\mathscr{B}$ of $\left(V, c^{\mathfrak{q}}\right)$. 
Assume first $x_{11112} \neq 0$ in $\mathscr{B}$. Then also $x_{12} \neq 0$. From Lemma 2.7 and the previous claim, we have a braided subspace

$$
W=\mathbb{k} x_{1}+\mathbb{k} x_{12}+\mathbb{k} x_{11112} \subset \mathcal{P}(\mathscr{B}),
$$

so Lemma 2.8 gives GK-dim $\mathscr{B}(W) \leq \mathrm{GK}$-dim $\mathscr{B}$. By a degree argument, $W$ has dimension three; from direct computation its Dynkin diagram is

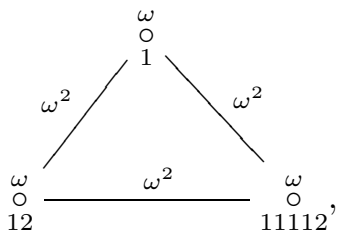

$$
\text { Cartan type }\left(\begin{array}{ccc}
2 & -1 & -1 \\
-1 & 2 & -1 \\
-1 & -1 & 2
\end{array}\right)
$$

Since the Cartan matrix is of affine type $A_{2}^{1}$, we have GK-dim $\mathscr{B}(W)=\infty$ by AAH2, Theorem 1.2(a)]. Thus GK-dim $\mathscr{B}=\infty$.

Assume now $x_{221} \neq 0$ in $\mathscr{B}$. Then $x_{21} \neq 0$, and since $q_{12} q_{21}=1$, we have $x_{12}=-q_{12} x_{21} \neq 0$. Consider $W^{\prime}=\mathbb{k} x_{1}+\mathbb{k} x_{12}+\mathbb{k} x_{221}$. We may now use the same argument as above. Indeed, $W^{\prime} \subset \mathcal{P}(\mathscr{B})$ has Dynkin diagram (3.6) replacing $x_{11112}$ by $x_{221}$, so GK-dim $\mathscr{B}\left(W^{\prime}\right)=\infty$ by the same reason as GK-dim $\mathscr{B}(W)=\infty$. Hence GK-dim $\mathscr{B}=\infty$. Thus $\breve{\mathscr{B}}_{\mathfrak{q}} \rightarrow \mathscr{B}$.

The verification of GK- $\operatorname{dim} \breve{\mathscr{B}}_{\mathfrak{q}}=6$ is postponed to Proposition 4.5.

3.3. A further reduction. Let $\mathscr{B}$ be a finite GK-dimensional pre-Nichols algebra of $\mathscr{B}_{\mathfrak{q}}$. We are naturally led to consider

$$
E:=\left\{(i, j): i \in \mathbb{I}^{3}, j \in \mathbb{I}^{1}, x_{i j} \neq 0 \text { in } \mathscr{B}\right\} .
$$

That is, $(i, j) \in E$ means that ord $q_{i i}=3, q_{j j}=1$ and $x_{i j} \neq 0$ in $\mathscr{B}$.

Remark 3.4. If $(i, j) \in E$, the braided vector space $\mathbb{k} x_{i} \oplus \mathbb{k} x_{i j} \subset \mathscr{B}$ is of Cartan type $A_{2}$ by Remark 3.1

Lemma 3.5. If $\left(i, j_{1}\right),\left(i, j_{2}\right) \in E$ then $j_{1}=j_{2}$.

Proof. Since $x_{i j_{1}}$ and $x_{i j_{2}}$ are $\mathbb{Z}^{\theta}$-homogeneous,

$$
c\left(x_{i j_{1}} \otimes x_{i j_{2}}\right)=\mathfrak{q}\left(\alpha_{i}+\alpha_{j_{1}}, \alpha_{i}+\alpha_{j_{2}}\right) x_{i j_{2}} \otimes x_{i j_{1}}=q_{i i} q_{i j_{2}} q_{j_{1} i} q_{j_{1} j_{2}} x_{i j_{2}} \otimes x_{i j_{1}} .
$$

Assume $j_{1} \neq j_{2}$. Then $x_{i}, x_{i j_{1}}$ and $x_{i j_{2}}$ have pairwise different $\mathbb{Z}^{\theta}$-degrees, so they span a 3 -dimensional braided subspace $W=\mathbb{k} x_{i}+\mathbb{k} x_{i j_{1}}+\mathbb{k} x_{i j_{2}} \subset \mathcal{P}(\mathscr{B})$. Now the Dynkin diagram of $W$ is

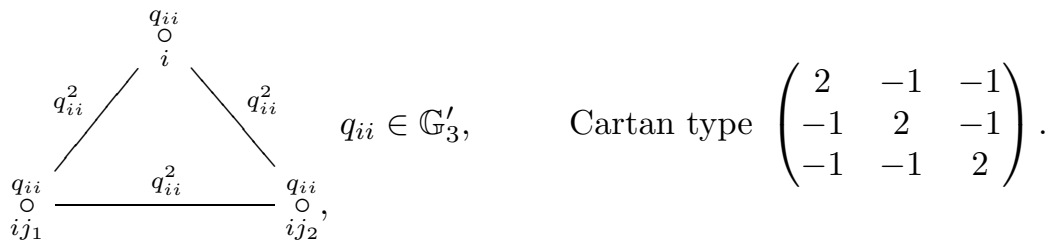

Since the Cartan matrix is of affine type $A_{2}^{(1)}$, we have GK-dim $\mathscr{B}(W)=\infty$ by AAH2, Theorem 1.2(a)]. Thus GK-dim $\mathscr{B}=\infty$, a contradiction. 


\section{Cartan type}

In this section we determine the finite GK-dimensional pre-Nichols algebras of braided vector spaces of finite Cartan type under some restrictions.

We fix a matrix $\mathfrak{q}=\left(q_{i j}\right)_{i, j \in \mathbb{I}}$ of non-zero scalars such that $q_{i i} \neq 1$ for all $i \in \mathbb{I}$ and a braided vector space $\left(V, c^{\mathfrak{q}}\right)$ with braiding given by $c^{\mathfrak{q}}\left(x_{i} \otimes x_{j}\right)=q_{i j} x_{j} \otimes x_{i}$, $i, j \in \mathbb{I}_{\theta}$, in a basis $\left\{x_{1}, \ldots, x_{\theta}\right\}$. Let $N_{i}=$ ord $q_{i i} \in \mathbb{N} \cup \infty$.

Recall that $\mathfrak{q}$, or $\left(V, c^{\mathfrak{q}}\right)$, is of Cartan type if there exists a Cartan matrix $\mathbf{a}=$ $\left(a_{i j}\right)_{i, j \in \mathbb{I}}$ such that $q_{i j} q_{j i}=q_{i i}^{a_{i j}}$ for all $i, j$. Let $i \in \mathbb{I}$. If $N_{i}=\infty$, then $a_{i j}$ are uniquely determined. Otherwise, we impose

$$
-N_{i}<a_{i j} \leq 0, \quad j \in \mathbb{I} .
$$

In this way we say that $\left(V, c^{\mathfrak{q}}\right)$, is of Cartan type $\mathbf{a}$.

We follow the terminology of $[\mathrm{K}]$. Cartan matrices are arranged in three families, namely: finite, affine and indefinite. We say that $\mathfrak{q}$, or $\left(V, c^{\mathfrak{q}}\right)$, belongs to one of these families if the corresponding a does.

In this section we assume that $\mathfrak{q}$ is of connected finite Cartan type and that $\operatorname{dim} \mathscr{B}_{\mathfrak{q}}<\infty$. Thus the possible Dynkin diagrams of $\mathfrak{q}$ have the following form, where $q$ is a root of unity in $\mathbb{k}$ of order $N>1$ :

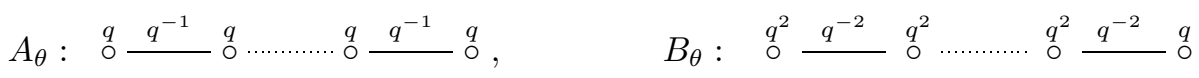

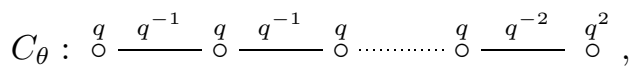

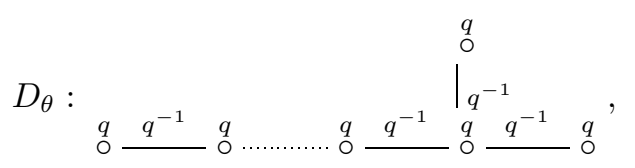

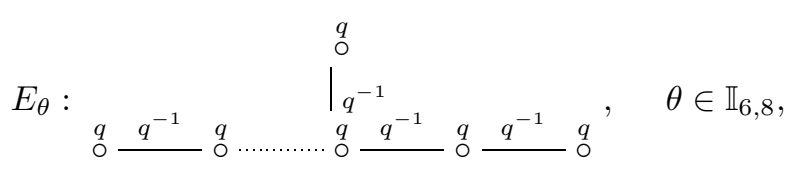

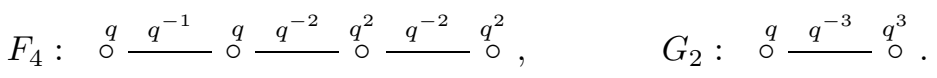

We refer to the survey AA2 for restrictions on $N$ and other features of $\mathscr{B}_{\mathfrak{q}}$ in each case. The quantum Serre relations are the following elements of $T(V)$ :

$$
\left(\operatorname{ad}_{c} x_{i}\right)^{1-a_{i j}} x_{j}, \quad i, j \in \mathbb{I}, i \neq j .
$$

By AS2, Lemma A.1] these are primitive in any pre-Nichols algebra. Let $\widetilde{\mathscr{B}}_{\mathfrak{q}}=$ $T(V) / \mathcal{I}_{\mathfrak{q}}$ be the distinguished pre-Nichols algebra of $\left(V, c^{\mathfrak{q}}\right)$, see $\$ 1.1 .3$.

Remark 4.1. From the detailed presentation in [AA2, §4] we see that the quantum Serre relations (4.2) generate $\mathcal{I}_{\mathfrak{q}}$ in the following cases:

- when a is of type $A_{2}$ or $B_{2}$ [AA2, pp. 397, 399, 400],

- when a is of type $G_{2}$ and $N \neq 4,6$ [AA2, pp. 410, 411],

- when a is simply-laced and $N>2$ [AA2, pp. 397, 404, 407],

- when a is of type B, C, or F and $N>4$ [AA2, pp. 399, 402, 409]. 
4.1. Quantum Serre relations. Let $\mathbf{a}=\left(a_{i j}\right)_{i, j \in \mathbb{I}}$ be a symmetrizable indecomposable generalized Cartan matrix and $\mathbf{d} \in \mathrm{GL}_{\theta}(\mathbb{Z})$ diagonal such that da is symmetric. The datum $(\mathbf{a}, \mathbf{d})$ is equivalent to an irreducible Cartan datum as in [Lu, 1.1.1] by setting

$$
\cdot: \mathbb{I} \times \mathbb{I} \rightarrow \mathbb{Z}, \quad i \cdot j=d_{i} a_{i j}, \quad i, j \in \mathbb{I} .
$$

Let $\mathfrak{g}=\mathfrak{g}(\mathbf{a})$ be the associated Kac-Moody algebra which has a triangular decomposition $\mathfrak{g}(\mathbf{a})=\mathfrak{g}^{+} \oplus \mathfrak{h} \oplus \mathfrak{g}^{-}$.

Let $q \in \mathbb{k}^{\times}$and consider the Dynkin diagram

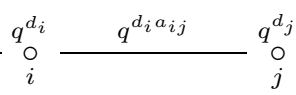

Let $\mathfrak{q}$ be any matrix with Dynkin diagram (4.3) and $\left(V, c^{\mathfrak{q}}\right)$ be the corresponding braided vector space with basis $\left(x_{i}\right)_{i \in \mathbb{I}}$. Notice that $\mathfrak{q}$ is of Cartan type but it is not necessarily of type a as (4.1) may not hold.

Let $\breve{\mathscr{B}}_{\mathfrak{q}}=T(V)$ modulo the ideal $\mathfrak{K}_{\mathfrak{q}}$ generated by the quantum Serre relations $\left(\operatorname{ad}_{c} x_{i}\right)^{1-a_{i j}}\left(x_{j}\right), i \neq j \in \mathbb{I}$, which is a pre-Nichols algebra of $V$.

Proposition 4.2. GK- $\operatorname{dim} \breve{\mathscr{B}}_{\mathfrak{q}} \geq \operatorname{dim} \mathfrak{g}^{+}$.

Proof. If $\xi \in \mathbb{k}, \xi^{2}=q$, then $\mathfrak{p}=\left(\xi^{d_{i} a_{i j}}\right)$ has Dynkin diagram (4.3). Let $\left(W, c^{\mathfrak{q}}\right)$ be the corresponding braided vector space with basis $\left(\widehat{x}_{i}\right)_{i \in \mathbb{I}}$.

Claim 1. GK-dim $\breve{\mathscr{B}}_{\mathfrak{q}}=$ GK-dim $\breve{\mathscr{B}}_{\mathfrak{p}}$.

Proof. By the proof of [AS3, Proposition 3.9] (or the proof of Lemma 2.9) there is a homogeneous linear isomorphism $\psi: T(V) \rightarrow T(W)$ determined by $\psi\left(x_{i}\right)=\widehat{x}_{i}$ for all $i \in \mathbb{I}$ and satisfying [AS3, Remarks 3.10]. Hence $\psi\left(\mathfrak{K}_{\mathfrak{q}}\right)=\mathfrak{K}_{\mathfrak{p}}$ and $\psi$ induces a homogeneous linear isomorphism $\psi: \breve{\mathscr{B}}_{\mathfrak{q}} \rightarrow \breve{\mathscr{B}}_{\mathfrak{p}}$. Then apply Lemma 2.1

Let now $\mathbf{f}$ be the $\mathbb{Q}(v)$-algebra defined in $[\mathrm{Lu}, 1.2 .5]$, where $v$ is an indeterminate and let ${ }_{\mathcal{A}} \mathbf{f}$ be the $\mathcal{A}:=\mathbb{Z}\left[v, v^{-1}\right]$-subalgebra spanned by the quantum divided powers of the generators of $\mathbf{f}[\mathrm{Lu}, 1.4 .7]$. By $[\mathrm{Lu}, 14.4 .3],{ }_{\mathcal{A}} \mathbf{f}$ is a free $\mathcal{A}$-module and

$$
P_{\mathcal{A}} \mathbf{f}=P_{\mathbf{f}} \text {. }
$$

Consider $\mathbb{k}$ as $\mathcal{A}$-module via $v \mapsto \xi$. Then we have the algebras ${ }_{\mathbb{k}} \mathbf{f}=\mathbb{k} \otimes_{\mathcal{A}} \mathbf{f}$ and ${ }_{\mathbb{k}} \tilde{\mathbf{f}}$ defined in [Lu, 33.1.1] (which is nothing else than $\breve{\mathscr{B}}_{\mathfrak{p}}$ ). By [Lu, 1.4.3], the quantum Serre relations hold in ${ }_{\mathbb{k}} \mathbf{f}$, hence we have a surjective algebra map $\breve{\mathscr{B}}_{\mathfrak{p}}={ }_{\mathbb{k}} \widetilde{\mathbf{f}} \rightarrow{ }_{\mathbb{k}} \mathbf{f}$. Thus

$$
\text { GK-dim } \breve{\mathscr{B}}_{\mathfrak{p}} \geq \mathrm{GK}-\operatorname{dim} \mathbf{f} \text {. }
$$

On the other hand, let $\mathbb{k}_{0}$ be $\mathbb{k}$ as $\mathcal{A}$-module via $v \mapsto 1$. Then $\mathbb{k}_{0} \widetilde{\mathbf{f}} \simeq U\left(\mathfrak{g}^{+}\right)$by [Lu, 33.1.1] and $\operatorname{dim}_{\mathbb{Q}(v)} \mathbf{f}_{\nu}=\operatorname{dim}_{\mathbb{k}_{0}}\left(\mathbb{k}_{0} \widetilde{\mathbf{f}}_{\nu}\right)$ by [Lu, 33.1.3]; that is

$$
\operatorname{GK}-\operatorname{dim} \mathbf{f}=\operatorname{GK}-\operatorname{dim}\left(\mathbb{k}_{0} \widetilde{\mathbf{f}}\right)=\operatorname{dim} \mathfrak{g}^{+},
$$

where the first equality holds by Lemma 2.1. The Proposition follows.

Example 4.3. Let $\mathbf{a}=\left(\begin{array}{cc}2 & -5 \\ -1 & 2\end{array}\right)$. Then (4.3) takes the form $\stackrel{q}{\circ} \underset{1}{q^{-5}} \stackrel{q^{5}}{\stackrel{0}{2}} \underset{2}{\text { with }}$ with $q \in \mathbb{k}^{\times}$. If $q_{12} \in \mathbb{k}^{\times}$and $q_{21}:=q_{12}^{-1} q^{-5}$, then $\mathfrak{q}=\left(\begin{array}{cc}q & q_{12} \\ q_{21} & q^{5}\end{array}\right)$ has the Dynkin 
diagram above. Here $\breve{\mathscr{B}}_{\mathfrak{q}}=\mathbb{k}\left\langle x_{1}, x_{2}\right\rangle$ modulo the relations

$$
\begin{aligned}
& x_{2}^{2} x_{1}-q_{21}(2)_{q} x_{2} x_{1} x_{2}+q q_{21}^{2} x_{1} x_{2}^{2}, \\
& x_{1}^{6} x_{2}-3 q_{12}^{2} x_{1}^{4} x_{2} x_{1}^{2}+3 q_{12}^{4} x_{1}^{2} x_{2} x_{1}^{4}-q_{12}^{6} x_{2} x_{1}^{6} .
\end{aligned}
$$

In this setting Proposition 4.2 gives GK-dim $\breve{\mathscr{B}}_{\mathfrak{q}}=\infty$.

Example 4.4. Let $\mathbf{a}=\left(\begin{array}{cc}2 & -3 \\ -1 & 2\end{array}\right)$. Then (4.3) takes the form $\stackrel{q}{\circ} \underset{1}{q^{-3}} \stackrel{q^{3}}{\stackrel{0}{2}} \underset{2}{2}$ with $q \in \mathbb{k}^{\times}$. If $q_{12} \in \mathbb{k}^{\times}$and $q_{21}:=q_{12}^{-1} q^{-3}$, then $\mathfrak{q}=\left(\begin{array}{cc}q & q_{12} \\ q_{21} & q^{3}\end{array}\right)$ has the Dynkin diagram above. Here $\breve{\mathscr{B}}_{\mathfrak{q}}=\mathbb{k}\left\langle x_{1}, x_{2}\right\rangle$ modulo the relations

$$
\begin{aligned}
& x_{2}^{2} x_{1}-q_{21}(2)_{q^{3}} x_{2} x_{1} x_{2}+q_{21}^{2} q^{3} x_{1} x_{2}^{2}, \\
& x_{1}^{4} x_{2}-q_{12}(4)_{q} x_{1}^{3} x_{2} x_{1}+q_{12}^{2} q\left(\begin{array}{l}
4 \\
2
\end{array}\right)_{q} x_{1}^{2} x_{2} x_{1}^{2}-q_{12}^{3}(4)_{q} x_{1}^{3} x_{2} x_{1}+q_{12}^{4} q^{6} x_{2} x_{1}^{4} .
\end{aligned}
$$

In this situation Proposition 4.2 establishes GK-dim $\breve{\mathscr{B}}_{\mathfrak{q}} \geq 6$.

This last example gains more relevance when the parameter $q \in \mathbb{k}^{\times}$specializes to a root of unity with small order.

Proposition 4.5. Let $\mathbf{a}$ and $q \in \mathbb{k}^{\times}$as in Example 4.4.

(a) If $q \in \mathbb{G}_{3}^{\prime}$ then GK-dim $\breve{\mathscr{B}}_{\mathfrak{q}}=6$.

(b) If $q \in \mathbb{G}_{2}^{\prime}$ then $x_{112}^{2}=0$ in $\breve{\mathscr{B}}_{\mathfrak{q}}$.

Proof. Let $q \in \mathbb{k}^{\times}$. Put $x_{1^{3} 2^{2}}=\left[x_{112}, x_{12}\right]$. By direct computation, in $\breve{\mathscr{B}}_{\mathfrak{q}}$ the following relations hold:

$$
\begin{aligned}
x_{12} x_{2} & =q_{12} q^{3} x_{2} x_{12}, \\
x_{112} x_{2} & =q_{12}^{2} q^{3} x_{2} x_{112}+q_{12} q^{2}(q-1)(2)_{q} x_{12}^{2}, \\
x_{1112} x_{2} & =q_{12}^{3} q^{3} x_{2} x_{1112}+q_{12} q\left(q^{2}-q-1\right) x_{1^{3} 2^{2}}+q_{12}^{2} q^{2}(q-1)(3)_{q} x_{12} x_{112}, \\
x_{1^{3} 2^{2}} x_{2} & =q_{12}^{3} q^{6} x_{2} x_{1^{3} 2^{2}}+q_{12}^{2} q^{3}(q-1)^{2}(2)_{q} x_{12}^{3}, \\
x_{1} x_{1^{3} 2^{2}} & =q_{12}^{2} q^{3} x_{1^{3} 2^{2}} x_{1}+x_{1112} x_{12}-q_{12}^{2} q^{3} x_{12} x_{1112}, \\
(2)_{q} x_{1112} x_{12} & =q_{12}^{2} q^{3}(2)_{q} x_{12} x_{1112}+q_{12} q(q-1)(3)_{q} x_{112}^{2} .
\end{aligned}
$$

(a) Here $q \in \mathbb{G}_{3}^{\prime}$, so the last relation above becomes

$$
x_{1112} x_{12}=q_{12}^{2} x_{12} x_{1112} .
$$

Substituting this in the penultimate equation we get

$$
x_{1} x_{1^{3} 2^{2}}=q_{12}^{2} x_{1^{3} 2^{2}} x_{1} .
$$

These equalities imply more commutations:

$$
\begin{aligned}
x_{11^{3} 2^{2}} x_{12} & =q_{12} x_{12} x_{1^{3} 2^{2}}, & x_{112} x_{1^{3} 2^{2}} & =q_{12} x_{1^{3} 2^{2}} x_{112}, \\
x_{1112} x_{112} & =q_{12} x_{112} x_{1112}, & x_{1112} x_{1^{3} 2^{2}} & =q_{12}^{3} x_{1^{3} 2^{2}} x_{1112} .
\end{aligned}
$$

Now we claim that $\breve{\mathscr{B}}_{\mathfrak{q}}$ is linearly spanned by

$$
\mathbb{B}=\left\{x_{2}^{n_{1}} x_{12}^{n_{2}} x_{1^{3} 2^{2}}^{n_{3}} x_{112}^{n_{4}} x_{1112}^{n_{5}} x_{1}^{n_{6}}: 0 \leq n_{1}, \ldots, n_{6}\right\} .
$$

Denote by $\mathcal{I}$ the linear span of $\mathbb{B}$. Since $1 \in \mathcal{I}$, it is enough to show that $\mathcal{I}$ is left ideal of $\breve{\mathscr{B}}_{\mathfrak{q}}$. If we multiply $x_{2}^{n_{1}} x_{12}^{n_{2}} x_{1^{3} 2^{2}}^{n_{3}} x_{112}^{n_{4}} x_{1112}^{n_{5}} x_{1}^{n_{6}}$ by $x_{1}$ on the left, we 
can use the previously deduced commutations between the (powers of the) $x_{\alpha}$ 's to successively rearrange the terms until we get a linear combination of elements in $\mathbb{B}$. The claim follows. Let $K=\left\{2,12,1^{3} 2^{2}, 112,1112,1\right\}$ be ordered by

$$
2>12>1^{3} 2^{2}>112>1112>1 \text {. }
$$

This order is convex, that is for $\alpha, \beta \in K$ with $\alpha>\beta$, the braided commutator $\left[x_{\alpha}, x_{\beta}\right]_{c}$ is a sum of monomials in the letters $\gamma$ such that $\beta<\gamma<\alpha$. Indeed this follows from the equalities above.

Consider next the lexicographical order on $\mathbb{B}$ induced by the order of $K$ and the corresponding $\mathbb{N}_{0}$-filtration $\mathcal{F}$ on $\breve{\mathscr{B}}_{\mathfrak{q}}$. Let $\operatorname{gr}_{\mathcal{F}}\left(\breve{\mathscr{B}}_{\mathfrak{q}}\right)$ be the associated graded algebra. By the convexity of the order, there is a natural projection from a quantum polynomial algebra $\mathbb{k}_{\mathrm{q}}\left[y_{1}, \ldots, y_{6}\right] \rightarrow \operatorname{gr}_{\mathcal{F}}\left(\breve{\mathscr{B}}_{\mathfrak{q}}\right)$, hence GK-dim $\operatorname{gr}_{\mathcal{F}}\left(\breve{\mathscr{B}}_{\mathfrak{q}}\right) \leq 6$. By [KL, Proposition 6.6] and Example 4.4 we also have GK-dim $\operatorname{gr}_{\mathcal{F}}\left(\breve{\mathscr{B}}_{\mathfrak{q}}\right)=\mathrm{GK}$-dim $\breve{\mathscr{B}}_{\mathfrak{q}} \geq 6$, so the equality holds.

(b) This follows by specialization at $q=-1$ in the relation

$$
(2)_{q} x_{1112} x_{12}=q_{12}^{2} q^{3}(2)_{q} x_{12} x_{1112}+q_{12} q(q-1)(3)_{q} x_{112}^{2} .
$$

Remark 4.6. Let us point out the relevance of (b). By Kharchenko's theory [Kh, $\breve{\mathscr{B}}_{\mathfrak{q}}$ has a PBW-basis. By Proposition 4.2 we know GK-dim $\breve{\mathscr{B}}_{\mathfrak{q}} \geq 6$ but, when $q \in \mathbb{G}_{2}^{\prime}$, the root $2 \alpha_{1}+\alpha_{2}$ will not contribute to GK-dim $\breve{\mathscr{B}}_{\mathfrak{q}}$ by (b) above. So even if $\mathbf{a}$ is of type $G_{2}$, one should not expect that the PBW generators are just those related to the six positive roots of $G_{2}$, as was the case in the proof of (a)

4.2. Type $A_{2}$. In this and the next subsections we seek eminent (families of) preNichols algebras in order to determine finite GK-dim pre-Nichols algebras of braidings of finite Cartan type. The distinguished pre-Nichols algebra will serve as the principal guide in our exploration.

4.2.1. Type $A_{2}$ with $N>3$.

Lemma 4.7. Assume a is of Cartan type $A_{2}$ with $N>3$. If $\mathscr{B}$ is a finite $G K$ dimensional pre-Nichols algebra of $\mathfrak{q}$, then $x_{112}=0$ and $x_{221}=0$ in $\mathscr{B}, i$. e. the distinguished pre-Nichols algebra $\widetilde{\mathscr{B}}_{\mathfrak{q}}$ is eminent, cf. Definition 2.3.

Proof. Assume $x_{i i j} \neq 0$ for some $i \neq j \in \mathbb{I}_{2}$; the 3 -dimensional braided subspace $W:=\mathbb{k} x_{j} \oplus \mathbb{k} x_{i} \oplus \mathbb{k} x_{i i j} \subset \mathcal{P}(\mathscr{B})$ has GK-dim $\mathscr{B}(W)<\infty$.

Consider the braided subspace $W_{1}=\mathbb{k} x_{i} \oplus \mathbb{k} x_{i i j} \subset W$. By direct computation, the braiding on $W_{1}$ is of Cartan type with the following Dynkin diagram and Cartan matrix:

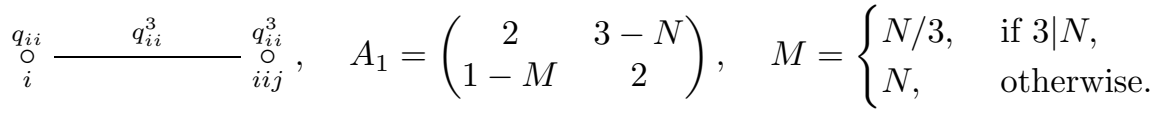

If either $N=5$ or $N>6$, it is evident that the Cartan matrix $A_{1}$ is not finite, so GK-dim $\mathscr{B}\left(W_{1}\right)=\infty$ by [AAH2, Theorem $\left.1.2(\mathrm{~b})\right]$. This contradicts GK-dim $\mathscr{B}<$ $\infty$.

For the remaining cases (i. e. $N=4$ and $N=6$ ), we consider the whole $W$. Since $\widetilde{\mathfrak{q}}\left(\alpha_{j}, 2 \alpha_{i}+\alpha_{j}\right)=\left(q_{j i} q_{i j}\right)^{2} q_{j j}^{2}=1$, the braiding on $W$ is of Cartan type with 
the following Dynkin diagram and Cartan matrix

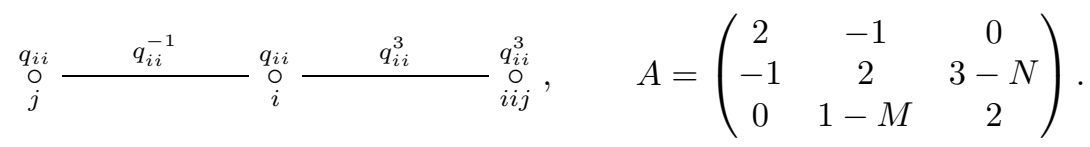

Now it is straightforward to verify that if $N=4$ or 6 , then $A$ is of affine type, which contradicts GK-dim $\mathscr{B}(W)<\infty$ by [AAH2, Theorem $1.2(\mathrm{~b})]$.

4.2.2. Type $A_{2}$ with $N=3$. Here is the first restriction.

Lemma 4.8. Assume $\mathbf{a}$ is of Cartan type $A_{2}$ with $N=3$. Let $\mathscr{B} \in \mathfrak{P r e}_{\mathrm{fGK}}$. Then $x_{i i i j}=0$ and $x_{j i i j}=0$ in $\mathscr{B}$ for all $i \neq j \in \mathbb{I}_{2}$.

Proof. Since $x_{i i j}$ is primitive, using that $\widetilde{\mathfrak{q}}\left(\alpha_{i}, 2 \alpha_{i}+\alpha_{j}\right)=q^{4} q^{-1}=1$ and $\widetilde{\mathfrak{q}}\left(\alpha_{j}, 2 \alpha_{i}+\right.$ $\left.\alpha_{j}\right)=q^{2} q^{-2}=1$, we get $x_{i i i j}, x_{j i i j} \in \mathcal{P}(\mathscr{B})$ by Lemma 2.7. Assume first $x_{i i i j} \neq 0$ in $\mathscr{B}$. The braided subspace $\mathbb{k} x_{i} \oplus \mathbb{k} x_{j} \oplus \mathbb{k} x_{i i i j} \subset \mathcal{P}(\mathscr{B})$ has finite GK-dim Nichols algebra. The Dynkin diagram is

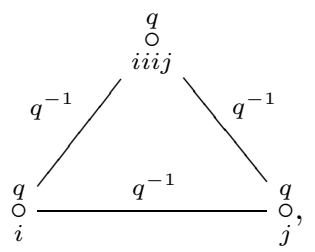

Cartan type $\left(\begin{array}{ccc}2 & -1 & -1 \\ -1 & 2 & -1 \\ -1 & -1 & 2\end{array}\right)$

The Cartan matrix is of affine type $A_{2}^{(1)}$, and by [AAH2, Theorem $1.2(\mathrm{a})$ ] this contradicts GK-dim $\mathscr{B}<\infty$.

If $x_{j i i j} \neq 0$, the same argument leads to a contradiction. Indeed, by direct computations, the Dynkin diagram of $U=\mathbb{k} x_{i} \oplus \mathbb{k} x_{j} \oplus \mathbb{k} x_{j i i j}$ is

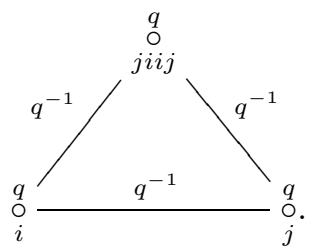

so GK- $\operatorname{dim} \mathscr{B}(U)=\infty$ by $\underline{\mathrm{AAH} 2}$, Theorem 1.2(a)].

Remark 4.9. Denote $\widehat{\mathscr{B}}=T(V) /\left\langle x_{1112}, x_{2221}, x_{2112}, x_{1221}\right\rangle$. The defining ideal of $\widehat{\mathscr{B}}$ is a Hopf ideal by the proof of Lemma 4.8. Let $\pi: \widehat{\mathscr{B}} \rightarrow \mathscr{B}(V)$ denote the natural projection. Let $\widehat{\mathcal{Z}}$ be the subalgebra of $\widehat{\mathscr{B}}$ generated by

$$
z_{1}:=x_{2}^{3}, \quad z_{2}:=x_{221}, \quad z_{3}:=x_{112}, \quad z_{4}:=x_{1}^{3}, \quad z_{5}:=x_{12}^{3} .
$$

The next results are devoted to prove that $\widehat{\mathscr{B}}$ is eminent.

\section{Lemma 4.10.}

(a) Given $i \neq j \in \mathbb{I}_{2}$, the following relations hold in $\widehat{\mathscr{B}}$ :

$$
\left[x_{i j}, x_{i i j}\right]_{c}=0=\left[x_{i j}, x_{j j i}\right]_{c} ; \quad\left[x_{i i j}, x_{j j i}\right]_{c}=0 .
$$

(b) $\widehat{\mathcal{Z}}$ is a normal braided Hopf subalgebra of $\widehat{\mathscr{B}}$.

(c) The $z_{i}$ 's q-commute; $B=\left\{z_{1}^{n_{1}} z_{2}^{n_{2}} z_{3}^{n_{3}} z_{4}^{n_{4}} z_{5}^{n_{5}}: n_{i} \in \mathbb{N}_{0}\right\}$ is a basis of $\widehat{\mathcal{Z}}$.

(d) $\widehat{\mathcal{Z}}=\operatorname{co} \pi \widehat{\mathscr{B}}$. 
Proof.

(a) Just compute using (2.8):

$$
\begin{aligned}
{\left[x_{i j}, x_{i i j}\right]_{c} } & =\left[x_{i},\left[x_{j}, x_{i i j}\right]_{c}\right]_{c}-q_{i j} x_{j}\left[x_{i}, x_{i i j}\right]_{c}+q_{j j} q_{j i}^{2}\left[x_{i}, x_{i i j}\right]_{c} x_{j}=0 ; \\
{\left[x_{i j}, x_{j j i}\right]_{c} } & =\left[x_{i},\left[x_{j}, x_{j j i}\right]_{c}\right]_{c}-q_{i j} x_{j}\left[x_{i}, x_{j j i}\right]_{c}+q_{i i} q_{i j}^{2}\left[x_{i}, x_{j j i}\right]_{c} x_{j}=0 ; \\
{\left[x_{i i j}, x_{j j i}\right]_{c} } & =\left[\left[x_{i}, x_{i j}\right]_{c}, x_{j j i}\right]_{c} \\
& =\left[x_{i},\left[x_{i j}, x_{j j i}\right]_{c}\right]_{c}-q_{i i} q_{i j} x_{i j}\left[x_{i}, x_{j j i}\right]_{c}+q_{i i}^{2} q_{i j}\left[x_{i}, x_{j j i}\right]_{c} x_{i j} \\
& =\left[x_{i},\left[x_{i j}, x_{j j i}\right]_{c}\right]_{c}=0 .
\end{aligned}
$$

(b) We claim that the generators of $\widehat{\mathcal{Z}}$ are annihilated by the braided adjoint action of $\widehat{\mathscr{B}}$. Fix $i \in \mathbb{I}_{2}$. By definition $\left(\operatorname{ad}_{c} x_{i}\right) z_{2}=0=\left(\operatorname{ad}_{c} x_{i}\right) z_{3}$. In $T(V)$ we have $\left(\operatorname{ad}_{c} x_{i}\right) x_{i}^{3}=x_{i}^{4}-q_{i i}^{3} x_{i}^{4}=0$, and if $j \neq i$ then

$$
\begin{aligned}
x_{j j j i}=\left(\operatorname{ad}_{c} x_{j}\right)^{3} x_{i} & =\sum_{k=0}^{3}(-1)^{k} q_{j i}^{k} q_{j j}^{k(k-1) / 2}\left(\begin{array}{l}
3 \\
k
\end{array}\right)_{q_{j j}} x_{j}^{3-k} x_{i} x_{j}^{k} \\
& =x_{j}^{3} x_{i}-q_{j i}^{3} x_{i} x_{j}^{3}=-q_{j i}^{3}\left(\operatorname{ad}_{c} x_{i}\right) x_{j}^{3} .
\end{aligned}
$$

Thus $\operatorname{ad}_{c} x_{i}$ annihilates $z_{1}$ and $z_{4}$. Finally, we proceed with $z_{5}$. From (a) we get the commutation $x_{112} x_{12}=q_{11}^{2} q_{12} x_{12} x_{112}$ in $\widehat{\mathscr{B}}$. Then using (2.6)

$$
\begin{aligned}
\left(\operatorname{ad}_{c} x_{1}\right) z_{5} & =x_{112} x_{12}^{2}+q_{11} q_{12} x_{12} x_{112} x_{12}+q_{11}^{2} q_{12}^{2} x_{12}^{2} x_{112} \\
& =q_{12}^{2}\left(q_{11}^{4}+q_{11}^{3}+q_{11}^{2}\right) x_{12}^{2} x_{112}=0 .
\end{aligned}
$$

For $\left(\operatorname{ad}_{c} x_{2}\right) z_{5}$, notice that on the one hand

$$
\begin{aligned}
{\left[x_{12},-\right]_{c}^{3} x_{2} } & =\sum_{k=0}^{3}(-1)^{k}\left(q_{12} q_{22}\right)^{k} q_{22}^{k(k-1) / 2}\left(\begin{array}{l}
3 \\
k
\end{array}\right)_{q_{22}} x_{12}^{3-k} x_{2} x_{12}^{k} \\
& =x_{12}^{3} x_{i}-q_{12}^{3} x_{i} x_{12}^{3}=-q_{12}^{3}\left(\operatorname{ad}_{c} x_{2}\right) x_{12}^{3} .
\end{aligned}
$$

On the other hand, using $\left[x_{12}, x_{2}\right]_{c}=q_{12}^{2} q_{22} x_{221}$ and (a) we get

$$
\begin{aligned}
{\left[x_{12},-\right]_{c}^{3} x_{2} } & =\left[x_{12},\left[x_{12},\left[x_{12}, x_{2}\right]_{c}\right]_{c}\right]_{c} \\
& =q_{12}^{2} q_{22}\left[x_{12},\left[x_{12}, x_{221}\right]_{c}\right]_{c}=\left[x_{12}, 0\right]_{c}=0,
\end{aligned}
$$

so $\left(\operatorname{ad}_{c} x_{2}\right) z_{5}=0$. This shows that $\widehat{\mathcal{Z}}$ is a normal subalgebra.

Next we verify that $\Delta\left(z_{i}\right) \in \widehat{\mathcal{Z}} \otimes \widehat{\mathcal{Z}}$ for $i \in \mathbb{I}_{5}$. This is clear for $i \in \mathbb{I}_{4}$, because those elements are primitive in $T(V)$; for $i=5$ we compute in $T(V)$ :

$$
\begin{aligned}
\Delta\left(x_{12}^{3}\right)= & x_{12}^{3} \otimes 1+1 \otimes x_{12}^{3} \\
& +\left(q^{-1}-q^{-2}\right) x_{112} \otimes x_{221}+\left(1-q^{-1}\right)^{3} q_{21}^{3} x_{1}^{3} \otimes x_{2}^{3} \\
& +(1-q)^{2} q_{21}^{3} x_{1112} \otimes x_{2}^{2}-\left(1-q^{-1}\right)^{2} q^{-1} x_{1}^{2} \otimes x_{2221} \\
& +(q-1) x_{1} \otimes\left[x_{12}, x_{221}\right]_{c}-\left(1-q^{-1}\right) q_{21}\left[x_{12}, x_{112}\right]_{c} \otimes x_{2} .
\end{aligned}
$$

Using (a) and the defining relations of $\widehat{\mathscr{B}}$ we see that $\widehat{\mathcal{Z}}$ is a Hopf subalgebra.

(c) We show that any pair of generators of $\widehat{\mathcal{Z}} q$-commute. By definition of $\widehat{\mathscr{B}}$, both $x_{1}$ and $x_{2} q$-commute with $z_{2}$ and $z_{3}$, so $z_{4}$ and $z_{1} q$-commute with $z_{2}$ and $z_{3}$. Secondly, (4.7) implies that $z_{1}$ and $z_{4} q$-commute. Thirdly, (a) shows that $z_{5} q$-commutes with $z_{3}$ and $z_{2}$, and also that $z_{2}$ and $z_{3} q$-commute. Lastly, $z_{5}$ 
$q$-commutes with $z_{4}$ by (4.8), and with $z_{1}$ by (4.9) and (4.10). Hence $B$ linearly generates $\widehat{\mathcal{Z}}$.

The linear independence is proven by steps.

Step 1 . The set $\left\{z_{1}^{n_{1}} z_{2}^{n_{2}} z_{3}^{n_{3}} z_{4}^{n_{4}}: n_{i} \in \mathbb{N}_{0}\right\}$ is linearly independent.

Proof. Consider the Hopf algebra $\widehat{\mathscr{B}} \# \mathbb{k} \mathbb{Z}^{2}$; let $A$ denote the subalgebra generated by $z_{1}, \ldots, z_{4}$ and $\mathbb{Z}^{2}$. Since all the generators of $A$ are either skew-primitives or grouplikes, it follows that $A$ itself is a pointed Hopf algebra. Notice that $z_{1}, \ldots, z_{4} \in \mathcal{P}(\widehat{\mathcal{Z}})$ are linearly independent. Indeed, they are non-zero because their $\mathbb{Z}$-degree is 3 , so they are linearly independent since their $\mathbb{Z}^{2}$-degrees are pairwise different (here we are using that the defining ideal of $\widehat{\mathscr{B}}$ is a Hopf ideal generated by $\mathbb{Z}^{2}$-homogeneous elements of $\mathbb{Z}$-degree 4 ). Hence the infinitesimal braiding of $A$ contains the braided vector space $\mathbb{k} z_{1} \oplus \cdots \oplus \mathbb{k} z_{4}$, which is quantum linear space with all points labeled by 1 . Thus $\left\{z_{1}^{n_{1}} z_{2}^{n_{2}} z_{3}^{n_{3}} z_{4}^{n_{4}} g: n_{i} \in \mathbb{N}_{0}, g \in \mathbb{Z}^{2}\right\} \subset A$ is linearly independent.

Step 2. The element $z_{5}$ does not belong to the left ideal $\widehat{\mathscr{B}}\left\langle z_{1}, z_{2}, z_{3}, z_{4}\right\rangle$.

Proof. We verify this using GAP.

The ideal $\widehat{\mathscr{B}}\left\langle z_{1}, z_{2}, z_{3}, z_{4}\right\rangle$ is a Hopf ideal because the generators are primitive. Denote the quotient by $R$ and consider the projection $\pi_{R}: \widehat{\mathscr{B}} \rightarrow R$.

Step 3. The set $\left\{\pi_{R}\left(z_{5}\right)^{n}: n \in \mathbb{N}_{0}\right\}$ is linearly independent.

Proof. Consider the Hopf algebra $R \# \mathbb{Z}^{2}$. The subalgebra generated by $\pi_{R}\left(z_{5}\right)$ and $\mathbb{Z}^{2}$ is a pointed Hopf algebra. Moreover, its infinitesimal braiding contains $\pi_{R}\left(z_{5}\right)$, which is a non-zero point by Step 2 and is labeled by 1 . Now proceed as in the proof of Step 1

Step 4 . We have $\left(\mathrm{id} \otimes \pi_{R}\right) \Delta\left(z_{5}^{n}\right)=\sum_{k=0}^{n}\left(\begin{array}{l}n \\ k\end{array}\right) z_{5}^{k} \otimes \pi_{R}\left(z_{5}\right)^{n-k}$ for all $n \in \mathbb{N}_{0}$.

Proof. The case $n=0$ is obvious, and $n=1$ follows from (4.11). An standard inductive argument for braided comultiplication yields the desired result.

Step 5 . The set $B$ is linearly independent.

Proof. Let $\sum_{n_{1}, \ldots, n_{5} \in \mathbb{N}_{0}} \lambda_{n_{1}, \ldots, n_{5}} z_{1}^{n_{1}} z_{2}^{n_{2}} z_{3}^{n_{3}} z_{4}^{n_{4}} z_{5}^{n_{5}}=0$. Assume there exists $n_{5}$ such that $\lambda_{n_{1}, \ldots, n_{5}} \neq 0$ for some $n_{1}, \ldots, n_{4} \in \mathbb{N}_{0}$; take $N$ as the maximal one. By Step 3 there is a linear map $f: R \rightarrow \mathbb{k}$ such that $f\left(\pi_{R}\left(z_{5}\right)^{n}\right)=\delta_{n, N}$ for all $n \in \mathbb{N}_{0}$. Now using Step 4 we compute

$$
\begin{aligned}
0 & =(\mathrm{id} \otimes f)\left(\mathrm{id} \otimes \pi_{R}\right) \Delta\left(\sum_{n_{1}, \ldots, n_{5} \in \mathbb{N}_{0}} \lambda_{n_{1}, \ldots, n_{5}} z_{1}^{n_{1}} z_{2}^{n_{2}} z_{3}^{n_{3}} z_{4}^{n_{4}} z_{5}^{n_{5}}\right) \\
& =\sum_{n_{1}, \ldots, n_{5} \in \mathbb{N}_{0}} \lambda_{n_{1}, \ldots, n_{5}}(\mathrm{id} \otimes f)\left(\mathrm{id} \otimes \pi_{R}\right)\left(\left(\prod_{i=1}^{4} \sum_{j=0}^{n_{i}}\left(\begin{array}{c}
n_{i} \\
j
\end{array}\right) z_{i}^{j} \otimes z_{i}^{n_{i}-j}\right) \Delta z_{5}^{n_{5}}\right) \\
& =\sum_{n_{1}, \ldots, n_{4} \in \mathbb{N}_{0}, n_{5} \leq N} \lambda_{n_{1}, \ldots, n_{5}}(\mathrm{id} \otimes f)\left(\sum_{j=0}^{n_{5}} z_{1}^{n_{1}} z_{2}^{n_{2}} z_{3}^{n_{3}} z_{4}^{n_{4}} z_{5}^{j} \otimes \pi_{R}\left(z_{5}^{n_{5}-j}\right)\right) \\
& =\sum_{n_{1}, \ldots, n_{4} \in \mathbb{N}_{0}} \lambda_{n_{1}, \ldots, n_{4}, N} z_{1}^{n_{1}} z_{2}^{n_{2}} z_{3}^{n_{3}} z_{4}^{n_{4}} \otimes 1 .
\end{aligned}
$$

This contradicts Step 1 . 
(d) Since $\Delta\left(z_{i}\right) \in \widehat{\mathcal{Z}} \otimes \widehat{\mathcal{Z}}$ and $\widehat{\mathcal{Z}}$ is normal, the right ideal $\widehat{\mathscr{B}} \widehat{\mathcal{Z}}^{+}$is a Hopf ideal. By $\mathrm{A}+$, Proposition 3.6 (c)] we get that the equality $\widehat{\mathcal{Z}}={ }^{\operatorname{co} \pi} \widehat{\mathscr{B}}$ is equivalent to $\mathscr{B}_{\mathfrak{q}} \simeq \widehat{\mathscr{B}} / \widehat{\mathscr{B}} \widehat{\mathcal{Z}}^{+}$. This last isomorphism holds because the diagram

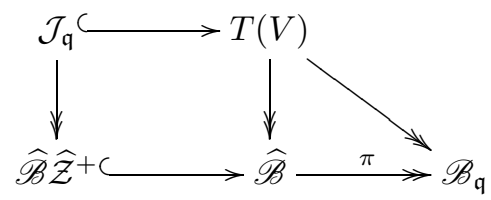

commutes.

\section{Proposition 4.11.}

(a) There is an extension of braided Hopf algebras

$$
\mathbb{k} \rightarrow \widehat{\mathcal{Z}} \hookrightarrow \widehat{\mathscr{B}} \rightarrow \mathscr{B}_{\mathfrak{q}} \rightarrow \mathbb{k} .
$$

(b) The pre-Nichols algebra $\widehat{\mathscr{B}}$ is eminent and GK-dim $\widehat{\mathscr{B}}=5$.

Proof.

(a) Follows from Lemma 4.10 (d).

(b) We know that $\widehat{\mathscr{B}}$ covers all elements of $\mathfrak{P r e}_{\mathrm{fGK}}$ by Lemma 4.8 , it remains to show that $\widehat{\mathscr{B}}$ itself belongs to $\mathfrak{P r e}_{\mathrm{fGK}}$. By A+, Proposition $\left.3.6(\mathrm{~d})\right]$ there is a right $\widehat{\mathcal{Z}}$-linear isomorphism $\mathscr{B}_{\mathfrak{q}} \otimes \widehat{\mathcal{Z}} \simeq \widehat{\mathscr{B}}$. Since $\mathscr{B}_{\mathfrak{q}}$ is finite dimensional, this implies that $\widehat{\mathscr{B}}$ is finitely generated as a $\widehat{\mathcal{Z}}$-module. Now [KL, Proposition 5.5] provides GK-dim $\widehat{\mathscr{B}}=\mathrm{GK}-\operatorname{dim} \widehat{\mathcal{Z}}=5$.

\subsection{Type $B_{2}$.}

Lemma 4.12. Assume that $\mathbf{a}$ is of Cartan type $B_{2}$. Then the distinguished preNichols algebra $\widetilde{\mathscr{B}}_{\mathfrak{q}}$ is eminent.

Proof. Here $N>2$. We may fix a braiding matrix $\mathfrak{q}$ such that $q_{11}=q_{22}^{2}$, so $q=q_{22}$ and $\widetilde{q_{12}}=q^{-2}$. Let $\mathscr{B}$ be a finite GK-dimensional pre-Nichols algebra of $V$. It is enough to prove that $x_{112}=0=x_{2221}$ in $\mathscr{B}$.

Assume first $x_{112} \neq 0$, and consider the 3-dimensional braided subspace $W:=$ $\mathbb{k} x_{1} \oplus \mathbb{k} x_{2} \oplus \mathbb{k} x_{112} \subset \mathcal{P}(\mathscr{B})$. Then GK-dim $\mathscr{B}(W)<\infty$ from Lemma 2.8. We split the proof according to the several possibilities for $N$.

$\varnothing N=3$. Now the braiding on $W$ is of Cartan type

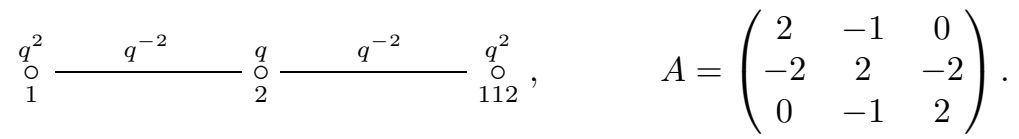

Since $A$ is of affine type $C_{2}^{(1)}$, this contradicts [AAH2, Theorem 1.2(a)].

$\curlyvee N=6$. In this case the braiding on $W_{2}:=\mathbb{k} x_{2} \oplus \mathbb{k} x_{112} \subset W$ is

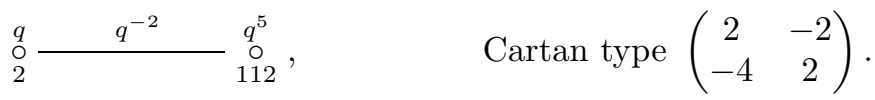

The Cartan matrix is of indefinite type, and by [AAH2, Theorem 1.2(b)] this contradicts GK-dim $\mathscr{B}(W)<\infty$. 
$\curlyvee N \neq 3,6$. The Dynkin diagram of $W_{1}:=\mathbb{k} x_{1} \oplus \mathbb{k} x_{112} \subset W$ is

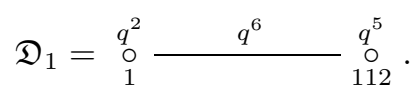

Since GK-dim $\mathscr{B}\left(W_{1}\right)<\infty$, it follows from [AAH2, Theorem 1.2(b)] that the associated root system is finite. Now $\mathfrak{D}_{1}$ is connected; by exhaustion on [H1, Table 1], we deduce that we must have $N=4$ or $N=8$. We turn again to $W_{2}$, whose Dynkin diagram is easily computed in each case:

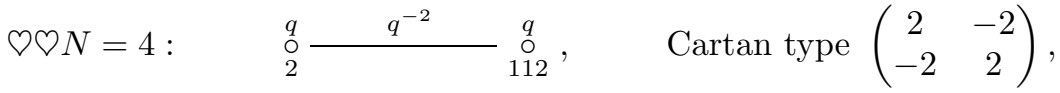

$$
\begin{aligned}
& \nabla \nabla N=8: \quad \quad \stackrel{q}{\circ} \quad \begin{array}{ll}
q^{-2} & \stackrel{q}{5}^{5} \\
112
\end{array}, \quad \text { Cartan type }\left(\begin{array}{cc}
2 & -2 \\
-2 & 2
\end{array}\right) \text {. }
\end{aligned}
$$

In any case the Cartan matrix is of affine type $A_{1}^{(1)}$, so GK-dim $\mathscr{B}\left(W_{2}\right)=\infty$ by AAH2, Theorem 1.2(b)].

Assume $x_{2221} \neq 0$ in $\mathscr{B}$. The subspace $U:=\mathbb{k} x_{1} \oplus \mathbb{k} x_{2} \oplus \mathbb{k} x_{2221} \subset \mathcal{P}(\mathscr{B})$ has dimension 3 and GK-dim $\mathscr{B}(U)<\infty$. Now $U_{1}:=\mathbb{k} x_{1} \oplus \mathbb{k} x_{2221} \subset U$ has connected Dynkin diagram

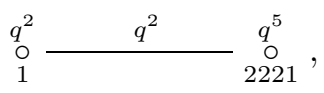

and it is finite by AAH2, Theorem 1.2(b)]. By exhaustion on [H1, Table 1] we deduce that $N=4$. Then the Dynkin diagram of $U$ is of Cartan type

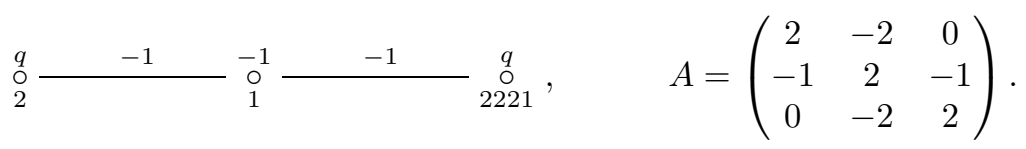

Since $A$ is of affine type $C_{2}^{(1)}$, this contradicts $\mathrm{AAH} 2$, Theorem 1.2(a)].

\subsection{Type $G_{2}$.}

Lemma 4.13. Assume that $\mathbf{a}$ is of Cartan type $G_{2}$. Then the quantum Serre relations hold in any $\mathscr{B} \in \mathfrak{P r e}_{\mathrm{fGK}}$. In particular, the distinguished pre-Nichols algebra $\widetilde{\mathscr{B}}_{\mathfrak{q}}$ is eminent if $N \neq 4,6$.

Proof. Here $N>3$. Let $\mathscr{B} \in \mathfrak{P r e}_{\mathrm{fGK}}(V)$; we show first that the quantum Serre relations $x_{11112}=0=x_{221}$ hold in $\mathscr{B}$.

Start assuming $x_{11112} \neq 0$. Then the 3 -dimensional subspace $W:=\mathbb{k} x_{1} \oplus \mathbb{k} x_{2} \oplus$ $\mathbb{k} x_{11112} \subset \mathcal{P}(\mathscr{B})$ satisfies GK-dim $\mathscr{B}(W) \leq \mathrm{GK}$-dim $\mathscr{B}$ by Lemma 2.8. The Dynkin diagram of $W_{1}:=\mathbb{k} x_{1} \oplus \mathbb{k} x_{11112} \subset W$ is

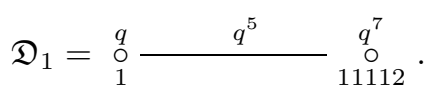

Since GK-dim $\mathscr{B}\left(W_{1}\right)<\infty$, it follows from [AAH2, Theorem 1.2(b)] that the root system of $\mathfrak{D}_{1}$ is finite. We split the proof according to the several possibilities for $N$.

$\varnothing N=5$. The diagram $\mathfrak{D}_{1}$ is disconnected, but we might consider instead $W_{2}:=\mathbb{k} x_{11112} \oplus \mathbb{k} x_{2} \subset W$, that satisfies GK-dim $\mathscr{B}\left(W_{2}\right)<\infty$ as well. By direct 
computation $W_{2}$ is of indefinite Cartan type:

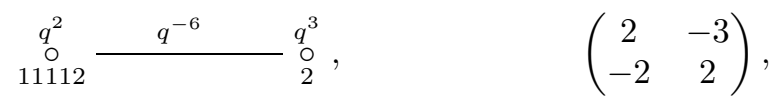

which is in contradiction with $\mathrm{AAH} 2$, Theorem 1.2(a)].

$\curlyvee N \neq 5$. Now $\mathfrak{D}_{1}$ is connected and finite; by inspection on [H1, Table 1], we must have $N=4$ or $N=6$.

$\varnothing \triangleright N=4$. In this case $W_{2}$ is of Cartan type

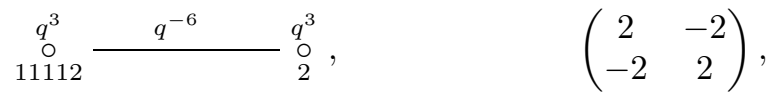

which is of affine type $A_{1}^{(1)}$, now contradicting [AAH2, Theorem 1.2(b)].

$\triangle \nabla N=6$. In this case the Dynkin diagram of $W$ is of Cartan type

$$
\stackrel{q}{\stackrel{q}{\circ}} \underset{1112}{\stackrel{q}{\circ}} \stackrel{q^{-1}}{1} \begin{aligned}
& q^{-3} \\
& \stackrel{q^{3}}{\circ}
\end{aligned}, \quad A=\left(\begin{array}{ccc}
2 & -1 & 0 \\
-1 & 2 & -3 \\
0 & -1 & 2
\end{array}\right) .
$$

By [AAH2, Theorem 1.2(b)] this contradicts GK-dim $\mathscr{B}(W)<\infty$, since $A$ is of affine type $G_{2}^{(1)}$.

Assume now $x_{221} \neq 0$ in $\mathscr{B}$. The subspace $U:=\mathbb{k} x_{1} \oplus \mathbb{k} x_{2} \oplus \mathbb{k} x_{221} \subset \mathcal{P}(\mathscr{B})$ has dimension 3 and GK-dim $\mathscr{B}(U)<\infty$. Consider two possibilities for $N$.

$\triangleright N \neq 4$. Now $U_{1}:=\mathbb{k} x_{1} \oplus \mathbb{k} x_{221} \subset U$ has connected Dynkin diagram

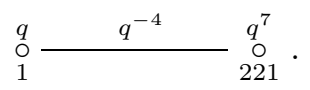

By exhaustion on [H1, Table 1] we conclude that this diagram is never finite, which contradicts AAH2, Theorem 1.2(b)], as GK-dim $\mathscr{B}\left(U_{1}\right)<\infty$.

$\nabla N=4$. In this case the braiding on $U$ is of Cartan type

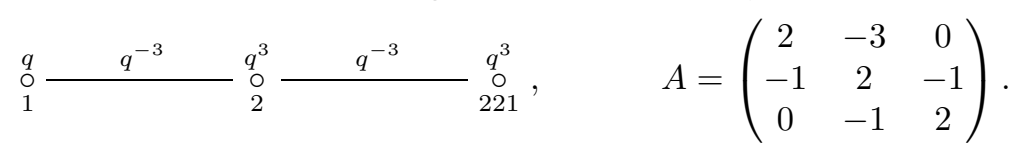

Since $A$ is of affine type $G_{2}^{(1)}$, this contradicts AAH2, Theorem 1.2(a)].

Thus the quantum Serre relations hold in $\mathscr{B}$. By Remark 4.1 this proves the assertion regarding $N \neq 4,6$.

\subsection{Type $A_{3}$.}

Lemma 4.14. If a is of Cartan type $A_{3}$ with $N>2$, then $\widetilde{\mathscr{B}}_{\mathfrak{q}}$ is eminent.

Proof. As $N>2$, the ideal $\mathcal{I}_{\mathfrak{q}}$ is generated by the quantum Serre relations $x_{13}=0$ and $x_{i i j}=0$ for $|j-i|=1$, cf. [AA2, p. 397]. Let $\mathscr{B} \in \mathfrak{P r e}_{\mathrm{fGK}}(\mathfrak{q})$. Then $x_{13}=0$ holds in $\mathscr{B}$ since the braided vector space $\mathbb{k} x_{1} \oplus \mathbb{k} x_{3}$ satisfies the hypothesis in Proposition 3.2

Turn to $x_{i i j}$ for some fix $i, j \in \mathbb{I}_{3}$ with $|j-i|=1$; in this case $\mathbb{k} x_{i} \oplus \mathbb{k} x_{j}$ is of Cartan type $A_{2}$. If $N>3$, then $x_{i i j}=0$ in $\mathscr{B}$ by Lemma 4.7. Only the case $N=3$ remains. Now we have $\mathfrak{q}\left(2 \alpha_{i}+\alpha_{j}, 2 \alpha_{i}+\alpha_{j}\right)=q_{i i}^{4}{\widetilde{q_{i j}}}^{2} q_{j j}=q^{5} q^{-2}=1$. Using AAH1, Lemma 2.8], in order to guarantee $x_{i i j}=0$ in $\mathscr{B}$ it is enough to find $k \in \mathbb{I}_{3}$ such that $\widetilde{\mathfrak{q}}\left(\alpha_{k}, 2 \alpha_{i}+\alpha_{j}\right) \neq 1$. It is straightforward to verify that the unique $k \in \mathbb{I}_{3}$ different from $i$ and $j$ does the trick. 


\subsection{Types $B_{3}$ and $C_{3}$.}

Lemma 4.15. The distinguished pre-Nichols algebra $\widetilde{\mathscr{B}}_{\mathfrak{q}}$ is eminent if either

(i) a is of type $B_{3}$, or

(ii) a is of type $C_{3}$.

Proof. Let $\mathscr{B} \in \mathfrak{P r e}_{\mathrm{fGK}}(\mathfrak{q})$. Then $x_{13}=0$ holds in $\mathscr{B}$. Indeed, the braided vector space $\mathbb{k} x_{1} \oplus \mathbb{k} x_{3}$ satisfies the hypothesis in Proposition 3.2 . Similarly, since $\mathbb{k} x_{2} \oplus \mathbb{k} x_{3}$ is of type $B_{2}$, it follows from Lemma 4.12 that the quantum Serre relations involving $x_{2}$ and $x_{3}$ hold in $\mathscr{B}$.

Step 1. If a is of Cartan type $B_{3}$, then the quantum Serre relations hold in any finite GK-dim pre-Nichols algebra.

Proof. Here $\mathbb{k} x_{1} \oplus \mathbb{k} x_{2}$ has Dynkin diagram $\stackrel{q^{2}}{\circ} \stackrel{q^{-2}}{q^{2}} \stackrel{q^{2}}{\circ}$, type $A_{2}$. Hence, if ord $q^{2}>$ 3 , we know from Lemma 4.7 that the quantum Serre relations between $x_{1}$ and $x_{2}$ hold in $\mathscr{B}$. Let us show that in the cases ord $q^{2}=2,3$ the same happens.

$\varnothing$ ord $q^{2}=2$. If $x_{112} \neq 0$ in $\mathscr{B}$, we get a subspace $\mathbb{k} x_{2} \oplus \mathbb{k} x_{3} \oplus \mathbb{k} x_{112} \subset \mathcal{P}(\mathscr{B})$ of dimension 3 with the following Dynkin diagram

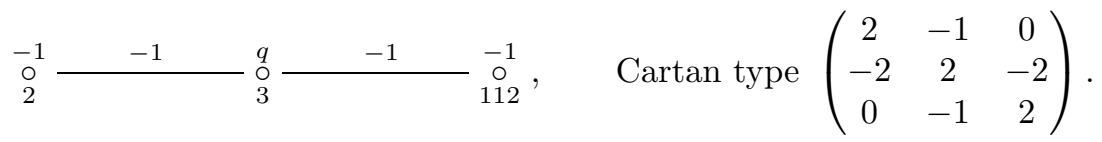

This matrix is of affine type $C_{2}^{(1)}$, hence GK- $\operatorname{dim} \mathscr{B}=\infty$, a contradiction.

Similarly, the assumption $x_{221} \neq 0$ yields a subspace of $\mathcal{P}(\mathscr{B})$ with braiding

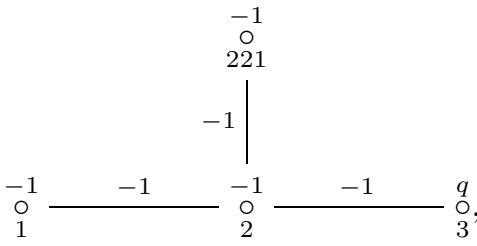

$$
\text { Cartan type }\left(\begin{array}{cccc}
2 & -1 & 0 & 0 \\
-1 & 2 & -1 & -1 \\
0 & -2 & 2 & 0 \\
0 & -1 & 0 & 2
\end{array}\right) \text {. }
$$

The Cartan matrix is of affine type $B_{3}^{(1)}$, and again $\operatorname{GK}-\operatorname{dim}(\mathscr{B})=\infty$.

$\varnothing$ ord $q^{2}=3$. Notice that

$$
\begin{aligned}
& \mathfrak{q}\left(2 \alpha_{1}+\alpha_{2}, 2 \alpha_{1}+\alpha_{2}\right)=q^{6}=1, \quad \widetilde{\mathfrak{q}}\left(2 \alpha_{1}+\alpha_{2}, \alpha_{3}\right)=q^{-2} \neq 1 ; \\
& \mathfrak{q}\left(\alpha_{1}+2 \alpha_{2}, \alpha_{1}+2 \alpha_{2}\right)=q^{6}=1, \quad \tilde{\mathfrak{q}}\left(\alpha_{1}+2 \alpha_{2}, \alpha_{3}\right)=q^{-4} \neq 1 .
\end{aligned}
$$

Assuming $x_{112} \neq 0$ in $\mathscr{B}$ we get $\mathbb{k} x_{3} \oplus \mathbb{k} x_{112} \subset \mathcal{P}(\mathscr{B})$ with Dynkin diagram $\stackrel{q}{q} q^{-2} \stackrel{1}{\circ}$. Then by [AAH1, Lemma 2.8] it follows that GK- $\operatorname{dim} \mathscr{B}=\infty$, a contradiction. By the same argument, we can not have $x_{221} \neq 0$ in $\mathscr{B}$.

The assertion (i) for $N>4$ follows since, in that case, $\widetilde{\mathscr{B}}_{\mathfrak{q}}$ is presented by the quantum Serre relations, cf. Remark 4.1 .

Step 2. If a is of Cartan type $B_{3}$ with $N=3$, then $\widetilde{\mathscr{B}}_{\mathfrak{q}}$ is eminent.

Proof. By [AA2, pp. 399, 400], $\widetilde{\mathscr{B}}_{\mathfrak{q}}$ is presented by the quantum Serre relations and $\left[x_{3321}, x_{32}\right]_{c}=0$. Given $\mathscr{B} \in \mathfrak{P r e}_{\mathrm{fGK}}$, let us show that $\left[x_{3321}, x_{32}\right]_{c} \in \mathcal{P}(\mathscr{B})$. Using 
$x_{13}=0$ a straightforward computation gives

$$
\begin{aligned}
\Delta\left(x_{3321}\right)= & x_{3321} \otimes 1+1 \otimes x_{3321}+\left(1-q_{33}\right) x_{332} \otimes x_{1} \\
& +\left(1-q_{33}\right) q_{33} x_{3} \otimes x_{321}+\left(1-q_{33}\right)\left(1-q_{22}\right) x_{3}^{2} \otimes x_{21} .
\end{aligned}
$$

With this we compute

$$
\begin{aligned}
\Delta\left(\left[x_{3321}, x_{32}\right]_{c}\right)= & {\left[x_{3321}, x_{32}\right]_{c} \otimes 1+1 \otimes\left[x_{3321}, x_{32}\right]_{c} } \\
& -\left(1-q_{33}\right)^{2}\left(1-q_{22}\right) q_{12} x_{3}^{2} \otimes x_{221} \\
& -\left(1-q_{33}\right) q_{12} q_{13} q_{23} q_{33} x_{3332} \otimes x_{21} \\
& +\left(1-q_{33}\right) q_{33}^{2} q_{12} q_{13} x_{332} \otimes\left(x_{321}-\left[x_{32}, x_{1}\right]_{c}\right) \\
& -\left(1-q_{33}\right) q_{23} q_{13} x_{33321} \otimes x_{2} \\
& +\left(1-q_{33}\right) q_{13} q_{12}\left[x_{332}, x_{32}\right]_{c} \otimes x_{1} \\
& +\left(1-q_{33}\right) q_{33} x_{3} \otimes\left(q_{13} q_{23} q_{33}\left[x_{3321}, x_{2}\right]_{c}+\left[x_{321}, x_{32}\right]_{c}\right)
\end{aligned}
$$

The third and fourth terms vanish in $\mathscr{B}$ by Step 1 . For the fifth term, a straightforward computation involving $x_{13}=0$ shows that $x_{321}=\left[x_{32}, x_{1}\right]_{c}$. The last three terms also vanish, but they require a more detailed analysis.

$\odot x_{33321}=0$ in $\mathscr{B}$. Notice that

$$
\begin{aligned}
\Delta\left(x_{33321}\right)= & x_{33321} \otimes 1+1 \otimes x_{33321} \\
& +\left(1-q_{33}\right) x_{3332} \otimes x_{1}-\left(1-q_{33}^{2}\right) q_{32} x_{332} \otimes x_{31},
\end{aligned}
$$

so this element is primitive in $\mathscr{B}$ by Step 1 Assuming $x_{33321} \neq 0$ we get a subspace $\mathbb{k} x_{1} \oplus \mathbb{k} x_{33321} \subset \mathcal{P}(\mathscr{B})$ where the braiding is given by

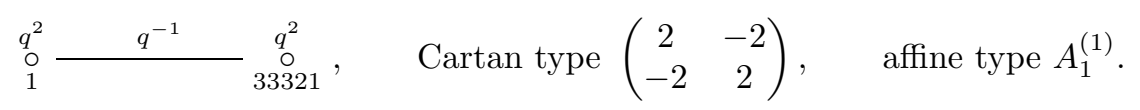

this contradicts GK-dim $\mathscr{B}<\infty$ by [AAH2, Theorem 1.2].

$\varnothing\left[x_{332}, x_{32}\right]_{c}=0$ in $\mathscr{B}$. Now we have

$$
\begin{aligned}
\Delta\left(\left[x_{332}, x_{32}\right]_{c}\right)= & {\left[x_{332}, x_{32}\right]_{c} \otimes 1+1 \otimes\left[x_{332}, x_{32}\right]_{c} } \\
& -\left(1-q_{33}\right)^{2} q_{23} x_{3}^{2} \otimes\left[x_{32}, x_{2}\right]_{c}-\left(1-q_{33}\right) q_{33} q_{23} x_{3332} \otimes x_{2} .
\end{aligned}
$$

The element $\left[x_{32}, x_{2}\right]_{c}$ is primitive in $\mathscr{B}$, so it vanishes by the same reason that $x_{223}$ does (cf. proof of Lemma 4.12). So $\left[x_{332}, x_{32}\right]_{c} \in \mathcal{P}(\mathscr{B})$ by Step 1 . If it is non-zero, consider $\mathbb{k} x_{1} \oplus \mathbb{k}\left[x_{332}, x_{32}\right]_{c} \subset \mathcal{P}(\mathscr{B})$ where the braiding is

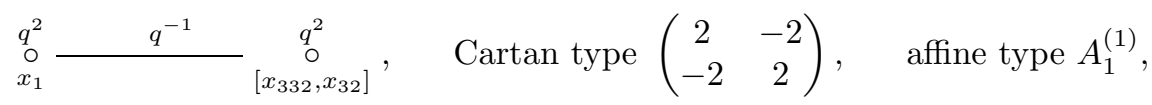

thus we get the same contradiction as with $x_{33321}$.

$\odot q_{13} q_{23} q_{33}\left[x_{3321}, x_{2}\right]_{c}+\left[x_{321}, x_{32}\right]_{c}=0$. Denote this element by $r$. Then

$$
\begin{aligned}
\Delta(r)= & r \otimes 1+1 \otimes r+\left(1-q_{33}\right) q_{22} q_{12} q_{13} x_{32} \otimes\left(x_{321}-\left[x_{32}, x_{1}\right]\right) \\
& +\left(1-q_{33}\right) q_{33} q_{12} q_{13} q_{23}\left[x_{3},\left[x_{32}, x_{2}\right]\right] \otimes x_{1} \\
& -\left(1-q_{33}\right) q_{22} q_{12} q_{13} q_{23} x_{3} \otimes\left[\left[x_{32}, x_{2}\right], x_{1}\right] .
\end{aligned}
$$


Since $\left[x_{32}, x_{2}\right]=0$ and $x_{321}-\left[x_{32}, x_{1}\right]=0$ in $\mathscr{B}$, it follows that $r$ is primitive. If $r \neq 0$ we consider $\mathbb{k} x_{2} \oplus \mathbb{k} r \subset \mathcal{P}(\mathscr{B})$. The Dynkin diagram is computed:

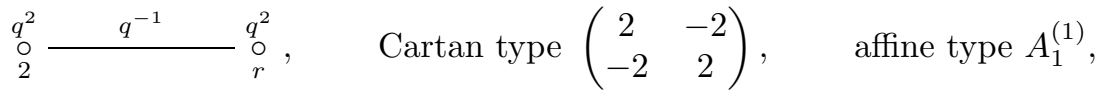

thus we get the same contradiction as before.

Using these three $\varnothing$ we get $\left[x_{3321}, x_{32}\right]_{c} \in \mathcal{P}(\mathscr{B})$. If this element is non-zero, consider $U=\mathbb{k} x_{3} \oplus \mathbb{k}\left[x_{3321}, x_{32}\right]_{c} \subset \mathcal{P}(\mathscr{B})$. We compute the braiding:

$$
\mathfrak{q}\left(\alpha_{1}+2 \alpha_{2}+3 \alpha_{3}, \alpha_{1}+2 \alpha_{2}+3 \alpha_{3}\right)=1, \quad \widetilde{\mathfrak{q}}\left(\alpha_{1}+2 \alpha_{2}+3 \alpha_{3}, \alpha_{3}\right)=q^{-1} \neq 1 .
$$

From AAH1, Lemma 2.8] it follows that GK-dim $\mathscr{B}(U)=\infty$, but this contradicts GK-dim $\mathscr{B}<\infty$. Then $\left[x_{3321}, x_{32}\right]_{c}=0$ in $\mathscr{B}$ and Step 2 holds.

Step 3. If a is of Cartan type $B_{3}$ with $N=4$, then $\widetilde{\mathscr{B}}_{\mathfrak{q}}$ is eminent.

Proof. By [AA2, pp. 399, 400], $\widetilde{\mathscr{B}}_{\mathfrak{q}}$ is presented by the quantum Serre relations and $\left[x_{123}, x_{2}\right]_{c}=0$. We claim that this element is primitive in any $\mathscr{B} \in \mathfrak{P r e}_{\mathrm{fGK}}$. Indeed, using that $x_{13}=0$ in $\mathscr{B}$, we get

$$
\begin{aligned}
\Delta\left(\left[x_{123}, x_{2}\right]_{c}\right)= & {\left[x_{123}, x_{2}\right]_{c} \otimes 1+1 \otimes\left[x_{123}, x_{2}\right]_{c} } \\
& -\left(1-\widetilde{q_{12}}\right) q_{32} x_{1} \otimes x_{223}+\left(1-\widetilde{q_{23}}\right) q_{32}\left[x_{12}, x_{2}\right]_{c} \otimes x_{3} .
\end{aligned}
$$

By straightforward computations, $\left[x_{12}, x_{2}\right]_{c} \in \mathcal{P}(\mathscr{B})$ and it vanishes by the same reason that $x_{221}$ does (cf. proof of Lemma 4.7). Since $x_{223}=0$, the claim follows.

Assume $\left[x_{123}, x_{2}\right]_{c} \neq 0$. Inside $\mathcal{P}(\mathscr{B})$ we have the 2 -dimensional subspace $U=$ $\mathbb{k} x_{3} \oplus \mathbb{k}\left[x_{123}, x_{2}\right]_{c}$ where the braiding is given by

$$
\stackrel{q}{\circ} \underset{x_{3}}{\stackrel{-1}{-q}} \begin{gathered}
-q \\
0 \\
{\left[x_{123}, x_{2}\right]}
\end{gathered}, \quad \text { Cartan type }\left(\begin{array}{cc}
2 & -2 \\
-2 & 2
\end{array}\right) .
$$

Since this matrix is of affine type $A_{1}^{(1)}$, from [AAH2, Theorem 1.2(b)] it follows GK-dim $\mathscr{B}(U)=\infty$, contradicting $\mathscr{B} \in \mathfrak{P r e}_{\mathrm{fGK}}$.

Step 4. If $\mathbf{a}$ is of Cartan type $C_{3}$, then the quantum Serre relations hold in any finite GK-dim pre-Nichols algebra.

Proof. Now $\mathbb{k} x_{1} \oplus \mathbb{k} x_{2}$ has Dynkin diagram $\stackrel{q}{\circ} \stackrel{q^{-1}}{ } \stackrel{q}{\circ}$, type $A_{2}$. If $N>3$, then the quantum Serre relations in $x_{1}$ and $x_{2}$ hold by Lemma 4.7. For the case $N=3$, let $i, j$ such that $\{i, j\}=\{1,2\}$ and suppose $x_{i i j} \neq 0$ in $\mathscr{B}$. Since $\mathfrak{q}\left(2 \alpha_{i}+\alpha_{j}, 2 \alpha_{i}+\right.$ $\left.\alpha_{j}\right)=q^{5} q^{-2}=1$ and $\widetilde{\mathfrak{q}}\left(2 \alpha_{i}+\alpha_{j}, \alpha_{3}\right)={\widetilde{q_{i 3}}}^{2} \widetilde{q_{j 3}} \neq 1$, we get GK-dim $\mathscr{B}=\infty$ by AAH1, Lemma 2.8].

The assertion (ii) for $N>4$ follows since, in that case, $\widetilde{\mathscr{B}}_{\mathfrak{q}}$ is presented by the quantum Serre relations, see Remark 4.1

Step 5. If a is of Cartan type $C_{3}$ with $N=3$, then $\widetilde{\mathscr{B}}_{\mathfrak{q}}$ is eminent.

Proof. Following [AA2, pp. 401, 402]) we see that $\widetilde{\mathscr{B}}_{\mathfrak{q}}$ is presented by the quantum Serre relations and $\left[\left[x_{123}, x_{2}\right]_{c}, x_{2}\right]_{c}=0$. Given $\mathscr{B} \in \mathfrak{P r e}_{\mathrm{fGK}}$, let us show that this 
element is primitive in $\mathscr{B}$. Using $x_{13}=0$ it follows that

$$
\begin{aligned}
\Delta\left(\left[x_{123}, x_{2}\right]_{c}\right)= & {\left[x_{123}, x_{2}\right]_{c} \otimes 1+1 \otimes\left[x_{123}, x_{2}\right]_{c}+\left(1-\widetilde{q_{12}}\right) x_{123} \otimes x_{2} } \\
& +\left(1-q^{2}\right) x_{12} \otimes x_{32}+\left(1-\widetilde{q_{12}}\right) x_{1} \otimes\left(x_{23} x_{2}-q_{32} x_{2} x_{23}\right) \\
& +\left(1-\widetilde{q_{23}}\right) q_{32}\left[x_{12}, x_{2}\right]_{c} \otimes x_{3} .
\end{aligned}
$$

By straightforward computations, $\left[x_{12}, x_{2}\right]_{c}=q_{12}^{2} q x_{221}$ in $T(V)$, and so $\left[x_{12}, x_{2}\right]_{c}$ vanishes in $\mathscr{B}$ by Step 4 . Then we obtain

$$
\begin{aligned}
& \Delta\left(\left[\left[x_{123}, x_{2}\right]_{c}, x_{2}\right]_{c}\right)=\left[\left[x_{123}, x_{2}\right]_{c}, x_{2}\right]_{c} \otimes 1+1 \otimes\left[\left[x_{123}, x_{2}\right]_{c}, x_{2}\right]_{c} \\
& \quad+\left(1-q^{2}\right) q_{32} q_{22}\left[x_{12}, x_{2}\right]_{c} \otimes x_{32}+\left(1-\widetilde{q_{12}}\right) q_{22} q_{32}^{2} x_{1} \otimes x_{2223},
\end{aligned}
$$

and now the claim follows from Step 4

If $\left[\left[x_{123}, x_{2}\right]_{c}, x_{2}\right]_{c} \neq 0$, consider $U=\mathbb{k} x_{1} \oplus \mathbb{k}\left[\left[x_{123}, x_{2}\right]_{c}, x_{2}\right]_{c} \subset \mathcal{P}(\mathscr{B})$. By AAH1, Lemma 2.8], since $\mathfrak{q}\left(\alpha_{1}+3 \alpha_{2}+\alpha_{3}, \alpha_{1}+3 \alpha_{2}+\alpha_{3}\right)=q^{12} q^{-9}=1$ and $\widetilde{\mathfrak{q}}\left(\alpha_{1}+3 \alpha_{2}+\alpha_{3}, \alpha_{1}\right)=q^{2} q^{-3} \neq 1$, we have GK-dim $\mathscr{B}(U)=\infty$. This contradicts $\mathscr{B} \in \mathfrak{P r e} \mathfrak{e}_{\mathrm{fGK}}$.

Step 6. If a is of Cartan type $C_{3}$ with $N=4$, then $\widetilde{\mathscr{B}}_{\mathfrak{q}}$ is eminent.

Proof. By [An1, Theorem 3.1], $\widetilde{\mathscr{B}}_{\mathfrak{q}}$ is presented by the quantum Serre relations and $\left[x_{123}, x_{23}\right]_{c}=0$. Let us show that this element is primitive in any pre-Nichols algebra $\mathscr{B}$ of finite GK-dim.

First we claim that $\left[x_{123}, x_{3}\right]_{c}=0$ in $\mathscr{B}$ : using that $x_{13}=0$ we compute

$$
\begin{aligned}
\Delta\left(\left[x_{123}, x_{3}\right]_{c}\right)= & {\left[x_{123}, x_{3}\right]_{c} \otimes 1+1 \otimes\left[x_{123}, x_{3}\right]_{c} } \\
& +\left(1-\widetilde{q_{12}} q_{23} q_{33}\right) x_{13} \otimes x_{23}+\left(1-\widetilde{q_{12}}\right) x_{1} \otimes\left[x_{23}, x_{3}\right]_{c} .
\end{aligned}
$$

Since $\left[x_{23}, x_{3}\right]_{c} \in \mathcal{P}(\mathscr{B})$, it vanishes in $\mathscr{B}$ by the same reason that $x_{332}$ does (cf. proof of Lemma 4.12). So $\left[x_{123}, x_{3}\right]_{c} \in \mathcal{P}(\mathscr{B})$. Hence, if it is non-zero we get a subspace $U=\mathbb{k} x_{1} \oplus\left[x_{123}, x_{3}\right]_{c} \subset \mathcal{P}(\mathscr{B})$ where the braiding is given by

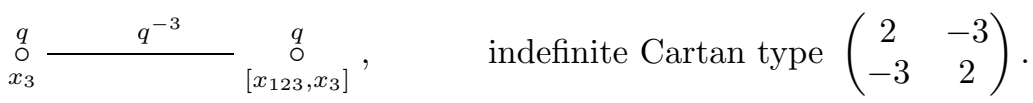

But then GK-dim $\mathscr{B}(U)=\infty$ by $[\mathrm{AAH} 2$, Theorem 1.2(b)], a contradiction.

Next we compute

$$
\begin{aligned}
& \Delta\left(\left[x_{123}, x_{23}\right]_{c}\right)=\left[x_{123}, x_{23}\right]_{c} \otimes 1+1 \otimes\left[x_{123}, x_{23}\right]_{c} \\
& \quad+\left(1-\widetilde{q_{23}}\right) q_{12} q_{22} q_{32} x_{2} \otimes\left[x_{123}, x_{3}\right]+\left(1-\widetilde{q_{23}}\right)^{2} q_{32} x_{1} \otimes\left[x_{12}, x_{2}\right]_{c} .
\end{aligned}
$$

Using the previous claim and the fact $\left[x_{12}, x_{2}\right]_{c}=q_{12}^{2} q x_{221}=0$ (by Step 4), we get $\left[x_{123}, x_{23}\right]_{c} \in \mathcal{P}(\mathscr{B})$. If $\left[x_{123}, x_{23}\right]_{c} \neq 0$, consider the subspace $W=\mathbb{k} x_{1} \oplus \mathbb{k} x_{2} \oplus$ $\mathbb{k}\left[x_{123}, x_{23}\right]_{c} \subset \mathcal{P}(\mathscr{B})$, where the braiding is

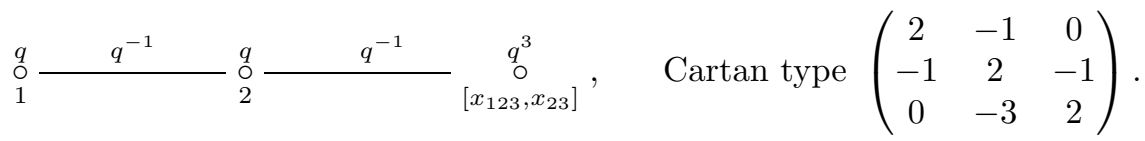

Since the Cartan matrix is of affine type $G_{2}^{(1)}$, it follows GK-dim $\mathscr{B}(W)=\infty$ by AAH2, Theorem 1.2(a)]. This contradicts $\mathscr{B} \in \mathfrak{P r e}_{\mathrm{fGK}}$.

The result follows. 
4.7. Some cases in rank $>3$. Here we assume that $\theta \geq 4$.

Lemma 4.16. In any of the following cases, $\widetilde{\mathscr{B}}_{\mathfrak{q}}$ is eminent.

(a) a is of Cartan type with simply laced Dynkin diagram and $N>2$.

(b) a is of type $B_{\theta}, C_{\theta}(\theta \geq 4)$ or $F_{4}$, and $N>4$.

Proof. By Remark 4.1 and the restrictions on $N, \widetilde{\mathscr{B}}_{\mathfrak{q}}$ is presented by the quantum Serre relations. Let $\mathscr{B} \in \mathfrak{P r e}_{\mathrm{fGK}}(\mathfrak{q})$. If $a_{i j}=0$, then $x_{i j}=0$ holds in $\mathscr{B}$ by Proposition 3.2. If $a_{i j} \neq 0$, then there is $k \in \mathbb{I}$ such that $\{i, j, k\}$ span a subdiagram of type $A_{3}, B_{3}$ or $C_{3}$. Then $\left(\operatorname{ad} x_{i}\right)^{1-a_{i j}}\left(x_{j}\right)=0$ by Lemmas 4.14 or 4.15 . Thus $\widetilde{\mathscr{B}}_{\mathfrak{q}} \rightarrow \mathscr{B}$.

In the next subsections we treat some remaining cases with small $N$.

4.8. Types $B_{\theta}, C_{\theta}, F_{4}, \theta>3, N=3,4$.

Lemma 4.17. If $\mathbf{a}$ is of types $B_{\theta}, C_{\theta}$, with $\theta>3$, or $F_{4}$, and $N=3$ or 4 , then $\widetilde{\mathscr{B}}_{\mathfrak{q}}$ is eminent.

Proof. We split the proof according to the type. Let $\mathscr{B} \in \mathfrak{P r} \mathfrak{r}_{\mathrm{fGK}}$.

$\curlyvee$ Type $F_{4}$. Here $\widetilde{\mathscr{B}}_{\mathfrak{q}}$ is presented by the quantum Serre relations and

$$
\left[x_{123}, x_{23}\right]_{c}=\left[x_{432}, x_{3}\right]_{c}=0 \text { if } N=4 ; \quad\left[x_{2234}, x_{23}\right]_{c}=0 \text { if } N=3 .
$$

Since $N>2$ we get $x_{14}=0$ in $\mathscr{B}$ from Proposition 3.2 . The subdiagram spanned by $\{1,2,3\}$ is of type $C_{3}$ thus the quantum Serre relations involving these indices hold in $\mathscr{B}$ by Lemma 4.15 (ii) Finally, $\{4,3,2\}$ span a diagram of type $B_{3}$ so the quantum Serre relations involving these indices hold in $\mathscr{B}$ by Lemma 4.15 (i). Moreover (4.12) are defining relations of the distinguished pre-Nichols algebra of type $B_{3}$ or $C_{3}$ for the corresponding $N$, hence Lemma 4.15 implies that these also vanish in $\mathscr{B}$.

$\curlyvee$ Type $B_{\theta}$. Here $\widetilde{\mathscr{B}}_{\mathfrak{q}}$ is presented by the quantum Serre relations and

$$
\left[x_{(i i+2)}, x_{i+1}\right]_{c}, i<\theta-1 \text {, if } N=4 ; \quad\left[x_{\theta \theta(\theta-1)(\theta-2)}, x_{\theta \theta-1}\right]_{c}, \quad \text { if } N=3 .
$$

The relations involving the indices $\{\theta-2, \theta-1, \theta\}$ hold in $\mathscr{B}$ by Lemma 4.15 (i) also $x_{i \theta}=0$ for any $i<\theta-1$ by Proposition 3.2 . We are left to treat the relations involving $\{1, \ldots, \theta-1\}$. If $N=3$ we only have the quantum Serre relations, which hold by Lemma 4.14. Turn to $N=4$. Now $\{1, \ldots, \theta-1\}$ form a subdiagram of type $A_{\theta-1}$ at a root of order 2 . If $\theta-1 \geq 4$ we apply Lemma 5.6 to get all the Serre relations except for $x_{221}$ and $x_{\theta-2 \theta-2 \theta-1}$. The last one holds by Lemma 4.15)(i) For the first one, we apply [AAH2, Theorem 1.2] since the diagram

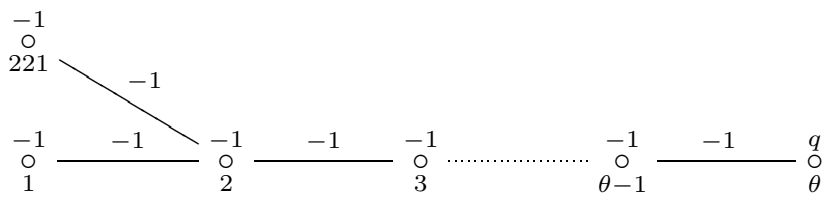

is of affine Cartan type. Now $\left[x_{(i i+2)}, x_{i+1}\right]_{c}=0$ for $i<\theta-2$ hold by Lemma 5.6 (e) We treat separately the last case standing.

D $\mathrm{Type} B_{4}$ with $N=4$. The relations $x_{221}$ and $x_{\theta-2 \theta-2 \theta-1}$ hold by the same reason as above. Moreover, we also have $x_{13}=0$. This follows from AAH1, Lemma 2.8] since $\mathfrak{q}\left(\alpha_{1}+\alpha_{3}, \alpha_{1}+\alpha_{3}\right)=1$ and $\widetilde{\mathfrak{q}}\left(\alpha_{1}+\alpha_{3}, \alpha_{4}\right) \neq 1$. Finally, using (5.1) and the relations deduced so far, we get that $\left[x_{(13)}, x_{2}\right]_{c}$ is primitive in $\mathscr{B}$. Notice that 
$\mathfrak{q}\left(\alpha_{1}+2 \alpha_{2}+\alpha_{3}, \alpha_{1}+2 \alpha_{2}+\alpha_{3}\right)=1$ and $\widetilde{\mathfrak{q}}\left(\alpha_{1}+2 \alpha_{2}+\alpha_{3}, \alpha_{4}\right) \neq 1$, so [AAH1, Lemma 2.8] applies again.

$\curlyvee$ Type $C_{\theta}$. Here $\widetilde{\mathscr{B}}_{\mathfrak{q}}$ is presented by the quantum Serre relations and

$$
\left[x_{(\theta-2 \theta)}, x_{\theta-1 \theta}\right]_{c}, \text { if } N=4 ; \quad\left[\left[x_{(\theta-2 \theta)}, x_{\theta-1}\right]_{c}, x_{\theta-1}\right], \text { if } N=3 .
$$

As before, Proposition 3.2 gives $x_{i \theta}=0$ for any $i<\theta-1$; all the relations involving the indices $\{\theta-2, \theta-1, \theta\}$ hold in $\mathscr{B}$ by Lemma 4.15 (ii) It remains to verify the relations involving $\{1, \ldots, \theta-1\}$. Here we only have the Serre relations. But these indices span a subdiagram of type $A_{\theta-1}, \theta-1 \geq 3$, at a root of unity of order 3 or 4, so they hold by Lemma 4.14.

4.9. Types $E_{6}, E_{7}$ and $E_{8}$ with $N=2$. By AA2, p. 407] the distinguished pre-Nichols algebra is presented by the quantum Serre relations and

$$
\left[x_{i j k}, x_{j}\right]_{c}=0 \text { if } i, j, k \text { are all different and } \widetilde{q_{i j}}, \widetilde{q_{j k}} \neq 1 \text {. }
$$

Lemma 4.18. Assume that Conjecture 2.6 is true. If a is of type $E_{6}, E_{7}$ or $E_{8}$ with $N=2$, then $\widetilde{\mathscr{B}}_{\mathfrak{q}}$ is eminent.

We point out that Conjecture 2.6 is needed only for a 5-dimensional braided vector space of indefinite Cartan type.

Proof. Let $\mathscr{B} \in \mathfrak{P r e}_{\mathrm{fGK}}(\mathfrak{q})$. First we deal with the quantum Serre relations, which are always primitive. Fix $i \neq j \in \mathbb{I}_{\theta}$. Consider two possibilities.

$\varnothing \widetilde{q_{i j}}=1$. In this case choose $k \in \mathbb{I}_{\theta}$ different from $i$ and $j$ such that $\widetilde{q}_{i}=1$ but $\widetilde{q_{i k}} \neq 1$. We get $\mathfrak{q}\left(\alpha_{i}+\alpha_{j}, \alpha_{i}+\alpha_{j}\right)=1$ and $\widetilde{\mathfrak{q}}\left(\alpha_{i}+\alpha_{j}, \alpha_{k}\right) \neq 1$. By AAH1, Lemma 2.8], this warranties $x_{i j}=0$ in $\mathscr{B}$.

$\varnothing \widetilde{q_{i j}} \neq 1$. In this case $i$ and $j$ are consecutive vertices in a subdiagram of type $A_{4}$ with $N=2$. By Lemma 5.6 (b) below, it follows that $x_{i i j}=0$ except in the following cases: $(i, j) \in\{(2,1),(\theta-3, \theta),(\theta-2, \theta-1)\}$. Fix such $(i, j)$, assume $x_{i i j} \neq 0$ and consider $\mathbb{k} x_{1} \oplus \cdots \oplus \mathbb{k} x_{\theta} \oplus \mathbb{k} x_{i i j} \subset \mathcal{P}(\mathscr{B})$. Then the Dynkin diagram of this braided vector space is of indefinite Cartan type. We illustrate the case $(i, j)=(\theta-3, \theta)$, the other cases being similar.

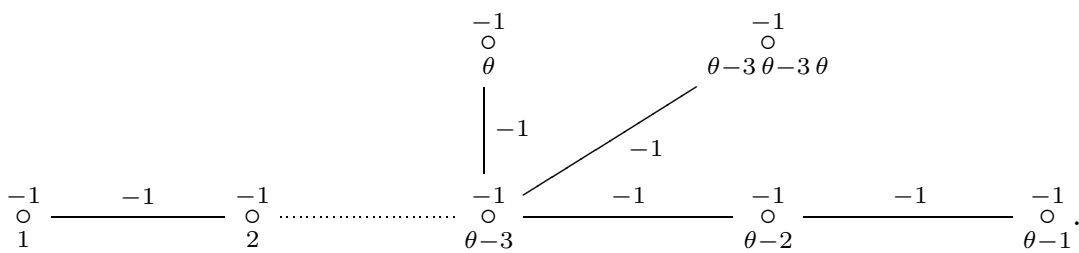

Thus Conjecture 2.6 and Lemma 2.8 imply GK-dim $\mathscr{B}=\infty$.

Finally, fix $i, j, k$ different such that $\widetilde{q_{i j}}, \widetilde{q_{j k}} \neq 1$. These are consecutive vertices in a suitable chosen subdiagram of type $A_{4}$. The Serre relations hold in $\mathscr{B}$, so by Lemma 5.6 (c) below we get that also $\left[x_{i j k}, x_{j}\right]_{c}=0$ in $\mathscr{B}$.

\section{ON THE OPEN CASES}

This section contain partial results towards those braidings of finite Cartan type which are still open. The detailed proofs can be found in $\underline{\mathbf{S}}$. 
5.1. Type $A_{2}$ with $N=2$.

Lemma 5.1. Assume a is of Cartan type $A_{2}$ with $N=2$. Let $\mathscr{B}$ be a finite GK-dimensional pre-Nichols algebra of $\mathfrak{q}$. The following hold:

(a) if $\mathscr{B} \in \mathfrak{P r} \mathfrak{Z}_{\mathrm{fGK}}^{\mathbb{Z}^{2}}$, then either $x_{112}=0$ or $x_{221}=0$ in $\mathscr{B}$;

(b) for different $i, j \in \mathbb{I}_{2},\left(\operatorname{ad}_{c} x_{i}\right)^{4} x_{j}=0$ in $\mathscr{B}$.

Question 5.2. Let $\widehat{\mathscr{B}}_{1}=\mathbb{k}\left\langle x_{1}, x_{2} \mid x_{221}, x_{11112}\right\rangle$. By Lemma 5.1 any $\mathscr{B} \in \mathfrak{P r e}_{\mathrm{fGK}}^{\mathbb{Z}^{2}}(V)$ is a quotient of either $\widehat{\mathscr{B}}_{1}$ or $\widehat{\mathscr{B}}_{2}:=\mathbb{k}\left\langle x_{1}, x_{2} \mid x_{112}, x_{22221}\right\rangle$. Clearly $\widehat{\mathscr{B}}_{1} \simeq \widehat{\mathscr{B}}_{2}$ as algebras. Is GK-dim $\widehat{\mathscr{B}}_{1}<\infty$ ?

5.2. Type $A_{3}$ with $N=2$.

Lemma 5.3. Assume a is of Cartan type $A_{3}$ with $N=2$. Let $\mathscr{B}$ be a finite $G K$-dimensional pre-Nichols algebra of $\mathfrak{q}$. Then the following hold in $\mathscr{B}$ :

(a) $x_{112}=0=x_{332}, x_{213} \stackrel{\star}{=} 0$,

(b) $x_{22221}=0=x_{22223}, x_{11113}=0=x_{33331}$,

(c) if $\mathscr{B} \in \mathfrak{P r e} \mathfrak{Z}_{\mathrm{fGK}}^{\mathbb{Z}^{3}}$, then at most one of $x_{113}, x_{331}, x_{221}, x_{223}$ is non-zero.

Remark 5.4. The relation $\star$ is relevant because in the tensor algebra

$$
\begin{array}{r}
\Delta\left(\left[x_{(13)}, x_{2}\right]_{c}\right)=\left[x_{(13)}, x_{2}\right] \otimes 1+1 \otimes\left[x_{(13)}, x_{2}\right]-2 q_{32} x_{1} \otimes x_{223} \\
-2 q_{12}^{2} q_{32} x_{221} \otimes x_{3}-2 q_{12}^{2} q_{32} x_{2} \otimes x_{213}+4 q_{12}^{2} q_{32} x_{2}^{2} \otimes x_{13} .
\end{array}
$$

Question 5.5. By Lemma 5.3 every $\mathscr{B} \in \mathfrak{P r} \mathfrak{e}_{\mathrm{fGK}}^{\mathbb{Z}^{3}}$ is covered by one of

$$
\begin{aligned}
& \widehat{\mathscr{B}}=\mathbb{k}\left\langle x_{1}, x_{2}, x_{3} \mid x_{112}, x_{332}, x_{22221}, x_{22223}, x_{11113}, x_{33331}, x_{213}\right\rangle, \\
& \quad \widehat{\mathscr{B}}_{1}=\widehat{\mathscr{B}} /\left\langle x_{113}\right\rangle, \quad \widehat{\mathscr{B}}_{2}=\widehat{\mathscr{B}} /\left\langle x_{331}\right\rangle, \quad \widehat{\mathscr{B}}_{3}=\widehat{\mathscr{B}} /\left\langle x_{221}\right\rangle, \quad \widehat{\mathscr{B}}_{4}=\widehat{\mathscr{B}} /\left\langle x_{223}\right\rangle .
\end{aligned}
$$

Are GK-dim $\widehat{\mathscr{B}}_{1}$ or GK-dim $\widehat{\mathscr{B}}_{3}<\infty$ ? ( $\widehat{\mathscr{B}}_{1} \simeq \widehat{\mathscr{B}}_{2}$ and $\widehat{\mathscr{B}}_{3} \simeq \widehat{\mathscr{B}}_{4}$ as algebras $)$.

5.3. Type $A_{\theta}, \theta \geq 4$ with $N=2$. In this setting $\widetilde{\mathscr{B}}_{\mathfrak{q}}$ is presented by

$$
x_{i j}=0, \quad|i-j|>1 ; \quad x_{i i j}=0, \quad|i-j|=1 ; \quad\left[x_{(i i+2)}, x_{i+1}\right]_{c}=0, \quad i \in \mathbb{I}_{\theta-2} .
$$

Lemma 5.6. Assume a is of Cartan type $A_{\theta}, \theta \geq 4$, with $N=2$. The following hold in any finite GK-dimensional pre-Nichols algebra $\mathscr{B}$ of $\mathfrak{q}$ :

(a) $x_{i j}=0$ for any $|i-j|>1$;

(b) $x_{i i j}=0$ for $|i-j|=1$ and $(i, j) \neq(2,1),(\theta-1, \theta)$;

(c) $x_{i i i i j}=0$ for $(i, j)=(2,1),(\theta-1, \theta)$;

(d) if $\mathscr{B} \in \mathfrak{P r e}_{\mathrm{fGK}}^{\mathbb{Z}^{\theta}}$, then either $x_{221}=0$ or $x_{\theta-1 \theta-1 \theta}=0$;

(e) if $(i, j) \in\{(2,1),(\theta-1, \theta)\}$ and $x_{i i j}=0$, then $\left[x_{(i-1 i+1)}, x_{i}\right]_{c}=0$.

Question 5.7. Let $\widehat{\mathscr{B}}_{1}$ denote the quotient of $T(V)$ by the relations

$$
\begin{aligned}
& x_{i j}=0, \quad|i-j|>1 \text {; } \\
& \left(\operatorname{ad}_{c} x_{\theta-1}\right)^{4} x_{\theta}=0 ; \\
& x_{i i j}=0, \quad|i-j|=1,(i, j) \neq(\theta-1, \theta) ; \\
& {\left[x_{(13)}, x_{2}\right]_{c}=0 \text {. }}
\end{aligned}
$$

Similarly, define $\widehat{\mathscr{B}}_{2}$ by the relations

$$
\begin{aligned}
x_{i j}=0, & |i-j|>1 ; & & \left(\operatorname{ad}_{c} x_{2}\right)^{4} x_{1}=0 ; \\
x_{i i j}=0, & |i-j|=1,(i, j) \neq(2,1) ; & & {\left[x_{(\theta-2 \theta)}, x_{\theta-1}\right]_{c}=0 . }
\end{aligned}
$$

(Clearly $\widehat{\mathscr{B}}_{2} \simeq \widehat{\mathscr{B}}_{1}$ as algebras). Is GK-dim $\widehat{\mathscr{B}}_{1}<\infty$ ? 
5.4. Type $D_{\theta}$ with $N=2$. Here (cf. [AA2, p. 404]) the distinguished pre-Nichols algebra $\widetilde{\mathscr{B}}_{\mathfrak{q}}$ is presented by the quantum Serre relations and a bunch of $q$-brackets coming from the several subdiagrams of type $A_{3}$, namely:

$$
\left[x_{(i i+2)}, x_{i+1}\right]_{c}, i \leq \theta-3 ; \quad\left[x_{\theta-3 \theta-2 \theta}, x_{\theta-2}\right]_{c} ; \quad\left[x_{\theta \theta-2 \theta-1}, x_{\theta-2}\right]_{c} .
$$

Lemma 5.8. Assume a is of Cartan type $D_{4}$ with $N=2$. The following relations hold in any $\mathscr{B} \in \mathfrak{P r e}_{\mathrm{fGK}}$ :

(a) if $i \neq j$ and $\widetilde{\mathfrak{q}}_{i j}=-1$, then $x_{i i j}=0$;

(b) if $i \neq j$ and $\widetilde{\mathfrak{q}}_{i j}=1$, then $x_{k i j}=0$ for all $k \in \mathbb{I}_{4}$;

(c) if $r$ is one of the elements in (5.2), then $\left(\operatorname{ad}_{c} x_{k}\right) r=0$ for all $k \in \mathbb{I}_{4}$.

Question 5.9. Let $\widehat{\mathscr{B}}$ denote the quotient of $T(V)$ by the relations (a) (b) and (c). Is GK-dim $\widehat{\mathscr{B}}<\infty$ ?

Lemma 5.10. Assume $\mathbf{a}$ is of Cartan type $D_{\theta}$ with $\theta>4$ and $N=2$. The following relations hold in any $\mathscr{B} \in \mathfrak{P r e}_{\mathrm{fGK}}(V)$ :

(a) all the defining relations of $\widetilde{\mathscr{B}}_{\mathfrak{q}}$ except $x_{\theta \theta-1}$ and $\left[x_{\theta \theta-2 \theta-1}, x_{\theta-2}\right]_{c}$;

(b) the relations $x_{k \theta \theta-1}$ and $\left(\operatorname{ad}_{c} x_{k}\right)\left[x_{\theta \theta-2 \theta-1}, x_{\theta-2}\right]_{c}$ for all $k \in \mathbb{I}_{\theta}$.

Question 5.11. Let $\widehat{\mathscr{B}}$ denote the quotient of $T(V)$ by the relations

$$
\begin{aligned}
x_{i j} & =0, \widetilde{\mathfrak{q}}_{i j}=1,(i, j) \neq(\theta, \theta-1) ; & & {\left[x_{\theta-3 \theta-2 \theta}, x_{\theta-2}\right]_{c}=0 ; } \\
x_{i i j} & =0, \widetilde{\mathfrak{q}}_{i j}=-1 ; & & {\left[x_{(i i+2)}, x_{i+1}\right]_{c}=0, i \leq \theta-3 ; } \\
x_{k \theta \theta-1} & =0, \quad k \in \mathbb{I}_{\theta} ; & & {\left[x_{k},\left[x_{\theta \theta-2 \theta-1}, x_{\theta-2}\right]\right]=0, k \in \mathbb{I}_{\theta} . }
\end{aligned}
$$

Is GK-dim $\widehat{\mathscr{B}}<\infty$ ? We conjecture that GK-dim $\widehat{\mathscr{B}}=\mathrm{GK}-\operatorname{dim} \widetilde{\mathscr{B}}_{\mathfrak{q}}+2$. This will be treated in a subsequent paper.

\section{ACKNOWLEDGMENTS}

We thank Iván Angiono and James Zhang for useful conversations. We are also grateful to the referee for detailed comments that helped to improve the presentation of the paper.

\section{REFERENCES}

[A] Nicolás Andruskiewitsch, An introduction to Nichols algebras, Quantization, geometry and noncommutative structures in mathematics and physics, Math. Phys. Stud., Springer, Cham, 2017, pp. 135-195. MR3751453

[AA1] Nicolás Andruskiewitsch and Iván Ezequiel Angiono, On Nichols algebras with generic braiding, Modules and comodules, Trends Math., Birkhäuser Verlag, Basel, 2008, pp. 4764, DOI 10.1007/978-3-7643-8742-6_3. MR2742620

[AA2] Nicolás Andruskiewitsch and Iván Angiono, On finite dimensional Nichols algebras of diagonal type, Bull. Math. Sci. 7 (2017), no. 3, 353-573, DOI 10.1007/s13373-017-0113-x. MR 3736568

[A+] Nicolás Andruskiewitsch, Iván Angiono, Agustín García Iglesias, Akira Masuoka, and Cristian Vay, Lifting via cocycle deformation, J. Pure Appl. Algebra 218 (2014), no. 4, 684-703, DOI 10.1016/j.jpaa.2013.08.008. MR3133699

[AAH1] N. Andruskiewitsch, I. Angiono, and I. Heckenberger, On finite GK-dimensional Nichols algebras over abelian groups, Mem. Amer. Math. Soc., to appear.

[AAH2] Nicolás Andruskiewitsch, Iván Angiono, and István Heckenberger, On finite GKdimensional Nichols algebras of diagonal type, Tensor categories and Hopf algebras, Contemp. Math., vol. 728, Amer. Math. Soc., Providence, RI, 2019, pp. 1-23, DOI 10.1090/conm/728/14653. MR3943743 
[AAH3] Nicolás Andruskiewitsch, Iván Angiono, and István Heckenberger, Liftings of Jordan and super Jordan planes, Proc. Edinb. Math. Soc. (2) 61 (2018), no. 3, 661-672, DOI 10.1017/s0013091517000402. MR3834727

[AAR] Nicolás Andruskiewitsch, Iván Angiono, and Fiorela Rossi Bertone, The quantum divided power algebra of a finite-dimensional Nichols algebra of diagonal type, Math. Res. Lett. 24 (2017), no. 3, 619-643, DOI 10.4310/MRL.2017.v24.n3.a2. MR3696597

[AS1] N. Andruskiewitsch and H.-J. Schneider, Lifting of quantum linear spaces and pointed Hopf algebras of order $p^{3}$, J. Algebra 209 (1998), no. 2, 658-691, DOI 10.1006/jabr.1998.7643. MR.1659895

[AS2] Nicolás Andruskiewitsch and Hans-Jürgen Schneider, Finite quantum groups and Cartan matrices, Adv. Math. 154 (2000), no. 1, 1-45, DOI 10.1006/aima.1999.1880. MR.1780094

[AS3] Eiichi Abe, Hopf algebras, Cambridge Tracts in Mathematics, vol. 74, Cambridge University Press, Cambridge-New York, 1980. Translated from the Japanese by Hisae Kinoshita and Hiroko Tanaka. MR.594432

[AS4] Nicolás Andruskiewitsch and Hans-Jürgen Schneider, On the classification of finitedimensional pointed Hopf algebras, Ann. of Math. (2) 171 (2010), no. 1, 375-417, DOI 10.4007/annals.2010.171.375. MR2630042

[An1] Iván Angiono, On Nichols algebras of diagonal type, J. Reine Angew. Math. 683 (2013), 189-251, DOI 10.1515/crelle-2011-0008. MR.3181554

[An2] Iván Ezequiel Angiono, A presentation by generators and relations of Nichols algebras of diagonal type and convex orders on root systems, J. Eur. Math. Soc. (JEMS) 17 (2015), no. 10, 2643-2671, DOI 10.4171/JEMS/567. MR3420518

[An3] Iván Angiono, Distinguished pre-Nichols algebras, Transform. Groups 21 (2016), no. 1, 1-33, DOI 10.1007/s00031-015-9341-x. MR3459702

[ACS] I. Angiono, E. Campagnolo, and G. Sanmarco, Finite GK-dimensional pre-Nichols algebras of super and standard type, arXiv:2009.04863

[B+] Mini-Workshop: infinite dimensional Hopf algebras, Oberwolfach Rep. 11 (2014), no. 2, 1111-1137, DOI 10.4171/OWR/2014/20. Abstracts from the mini-workshop held April 13-19, 2014; Organized by Ken Brown, Ken Goodearl, Tom Lenagan, and James Zhang. MR 3379364

[DKP] C. De Concini, V. G. Kac, and C. Procesi, Quantum coadjoint action, J. Amer. Math. Soc. 5 (1992), no. 1, 151-189, DOI 10.2307/2152754. MR.1124981

[GAP] The GAP Group, GAP - Groups, Algorithms and Programming, Version 4.9 .3 (2018), http://www.gap-system.org.

[G] K. R. Goodearl, Noetherian Hopf algebras, Glasg. Math. J. 55 (2013), no. A, 75-87, DOI 10.1017/S0017089513000517. MR3110805

[H1] I. Heckenberger, Classification of arithmetic root systems, Adv. Math. 220 (2009), no. 1, 59-124, DOI 10.1016/j.aim.2008.08.005. MR2462836

[H2] I. Heckenberger, Lusztig isomorphisms for Drinfel'd doubles of bosonizations of Nichols algebras of diagonal type, J. Algebra 323 (2010), no. 8, 2130-2182, DOI 10.1016/j.jalgebra.2010.02.013. MR2596372

[K] Victor G. Kac, Infinite-dimensional Lie algebras, 3rd ed., Cambridge University Press, Cambridge, 1990. MR 1104219

[Kh] Vladislav Kharchenko, Quantum Lie theory: A multilinear approach, Lecture Notes in Mathematics, vol. 2150, Springer, Cham, 2015. MR3445175

[KL] Günter R. Krause and Thomas H. Lenagan, Growth of algebras and Gelfand-Kirillov dimension, Revised edition, Graduate Studies in Mathematics, vol. 22, American Mathematical Society, Providence, RI, 2000. MR1721834

[L] Gongxiang Liu, A classification result on prime Hopf algebras of GK-dimension one, J. Algebra 547 (2020), 579-667, DOI 10.1016/j.jalgebra.2019.12.003. MR4042591

[Lu] George Lusztig, Introduction to quantum groups, Progress in Mathematics, vol. 110, Birkhäuser Boston, Inc., Boston, MA, 1993. MR1227098

[R] Marc Rosso, Quantum groups and quantum shuffles, Invent. Math. 133 (1998), no. 2, 399-416, DOI 10.1007/s002220050249. MR.1632802

[S] G. Sanmarco, Aportes a la clasificación de álgebras de Hopf punteadas de dimensión de Gelfand-Kirillov finita, Tesis doctoral, FaMAF, Un. Nac. Córdoba (2020).

[Sw] Moss E. Sweedler, Hopf algebras, Mathematics Lecture Note Series, W. A. Benjamin, Inc., New York, 1969. MR0252485 
[T] Mitsuhiro Takeuchi, Survey of braided Hopf algebras, New trends in Hopf algebra theory (La Falda, 1999), Contemp. Math., vol. 267, Amer. Math. Soc., Providence, RI, 2000, pp. 301-323, DOI 10.1090/conm/267/04277. MR1800719

Facultad de Matemática, Astronomía y Física, Universidad nacional de Córdoba. Ciem - Conicet. Medina Allende S/n (5000) Ciudad Universitaria, Córdoba, ArGENTINA

Email address: andrus@famaf.unc.edu.ar

Facultad de Matemática, Astronomía y Física, Universidad nacional de Córdoba. Ciem - COnicet. Medina Allende S/N (5000) Ciudad Universitaria, Córdoba, ArGENTINA

Email address: gsanmarco@famaf.unc.edu.ar 